\author{
Final Report \\ For \\ Department of Energy \\ under \\ Phase I SBIR Contract No. DE-FG02-03ER83690

\section{Characterization of Solid Oxide Fuel Cell Components \\ Using Electromagnetic Model-Based Sensors}

\author{
Prepared for \\ Lane C. Wilson, Ph.D. \\ National Energy Technology Laboratory \\ U.S. Department of Energy \\ 3610 Cochrans Mill Road \\ Morgantown, WV 26507
}

Submitted by

JENTEK Sensors, Inc.

110-1 Clematis Avenue

Waltham, MA 02453

September 2004 


\section{Table of Contents}

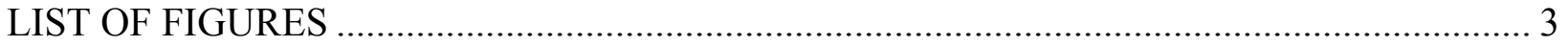

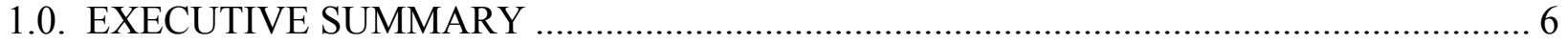

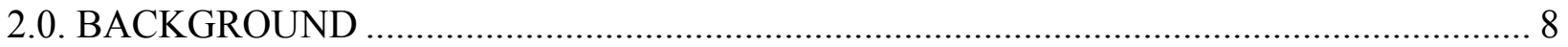

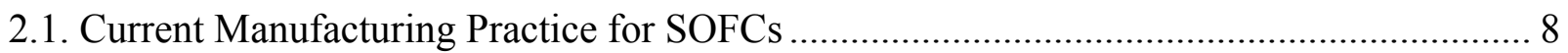

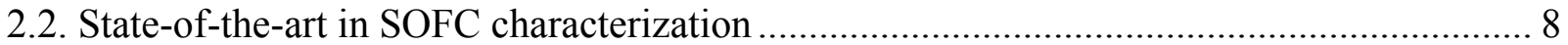

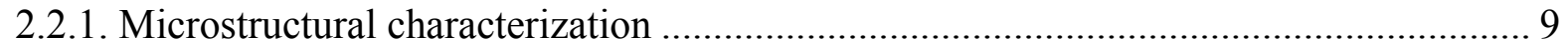

2.2.2. Permeability and diffusivity characterization. ......................................................... 9

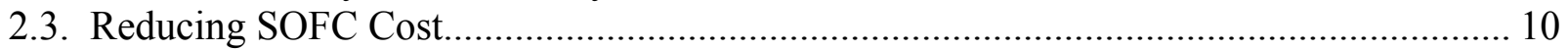

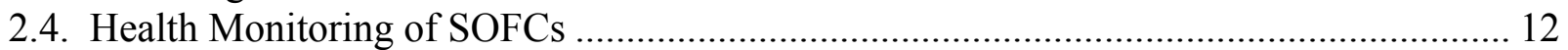

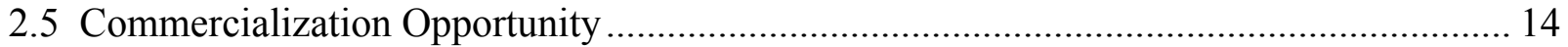

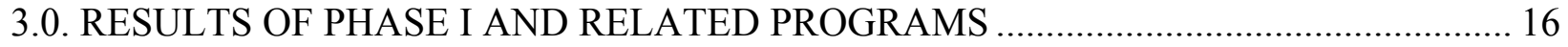

3.1. Summary of Phase I Accomplishments .............................................................. 16

3.2. Model-based Sensors, Description of Technology …………………………................... 17

3.2.1. The Meandering Winding Magnetometer (MWM) ……………………………..... 17

3.2.2. Interdigitated Electrode Dielectrometer (IDED) ......................................................... 20

3.2.3. Measurement Grid-Based Inversion Methods............................................................ 22

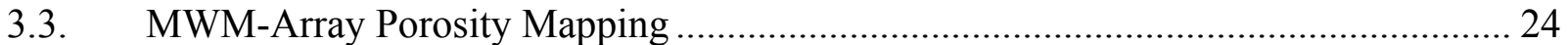

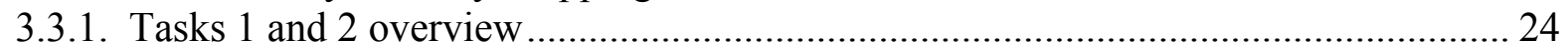

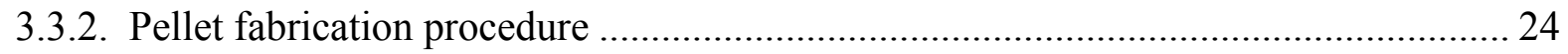

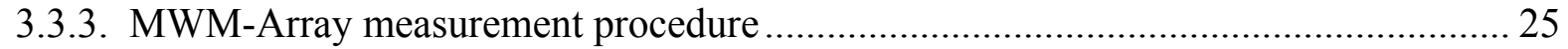

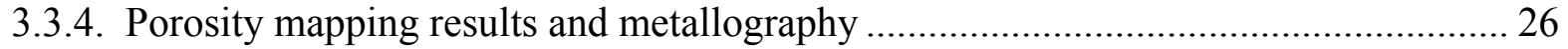

3.4. MWM Stress Measurements in Ferromagnetic Materials ......................................... 29

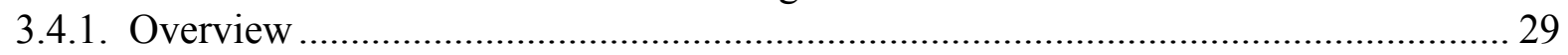

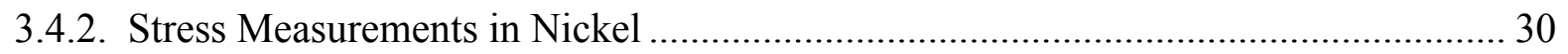

3.4.3. Determination of Ni permeability and optimization of lift-off .................................... 33

3.4.4. Scanning Ni plate to map magnetic permeability across its surface ............................ 33

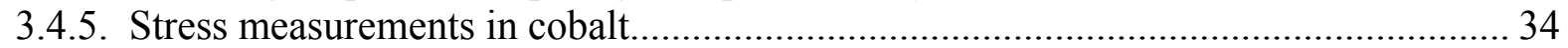

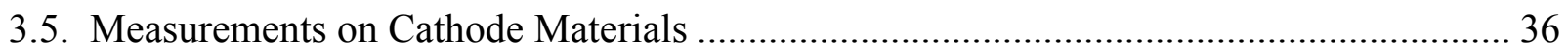

3.6. Identification of Another Property/Application of Interest .............................................. 42

3.7. Monitoring and Control of SOFC Components with Model-based Sensors .................... 43

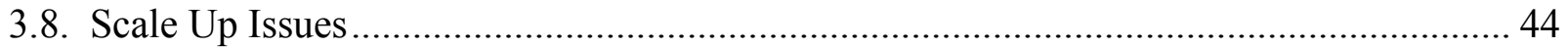

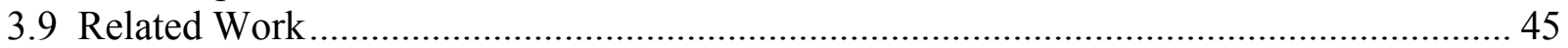

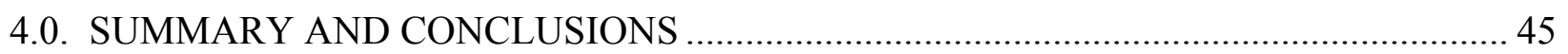

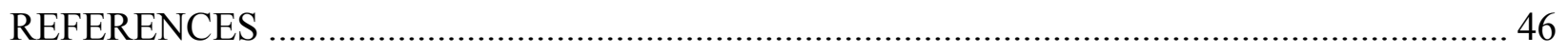




\section{LIST OF FIGURES}

Figure 1. SOFC stack cost vs. percentage of defective Electrode/Electrolyte Assemblies (EEAs).

Figure 2. SOFC stack cost vs. power density.

Figure 3. Adaptive damage tolerance flow chart.

Figure 4. (a) Original MWM sensor construct, (b) enhanced MWM design, (c) an MWM-Array design, (d) segmented field MWM sensor.

Figure 5. (a) Interdigitated electrode (IDED) sensor in contact with a test material; and (b) commercially available JENTEK sensor and enclosure for capacitive sensing of dielectric samples.

Figure 6. (a) A three-wavelength sensor with three separate sensing regions of different wavelength formed on a Teflon substrate with wavelengths of $5 \mathrm{~mm}, 2.5 \mathrm{~mm}$, and $1 \mathrm{~mm}$. (b) Photograph and corresponding schematic for a two-wavelength co-located interdigitated electrode sensor.

Figure 7. Multiple co-located dielectric sensors sense the same region of the test material, ensuring that the longer and shorter wavelengths have the same average lift-off or air gap thickness.

Figure 8. Segmented-field IDED sensor with rotational symmetry.

Figure 9. $630 \mathrm{kHz}$ conductivity lift-off grid generated for conductivities of 2-25\%IACS and liftoff values of 2 to 32 mils. Grids are used to rapidly convert sensor response into material property values.

Figure 10. Dielectric constant/lift-off measurement grid and results of two-unknown measurements carried out with the circular IDED shown in Figure 10.

Figure 11. Flow chart of grid (sensor response database) generation process.

Figure 12. Pellet 3 on the left is Ni/YSZ of standard $25-30 \%$ porosity. Pellet 1 on the right is $\mathrm{Ni} /$ YSZ with substantially greater porosity. Both pellets are approximately $27 \mathrm{~mm}$ (1.05 in.) dia. $\times 1.3 \mathrm{~mm}(0.05 \mathrm{in}$.) thick.

Figure 13. Two pellet sections that were also examined in this study. On the left is Ni/YSZ and on the right is Ni/GDC. Both specimens are approximately $1.1 \mathrm{~mm}(0.050 \mathrm{in}$.) thick.

Figure 14. Schematic of sensor/probe assembly (top), and picture of probe/sensor assembly mounted in cart (bottom). Cart was manually rolled across pellet, and encoder mounted in front wheel of cart recorded measurement position as sensor was scanned across pellet. 
Figure 15. Scan procedure for measuring Ni/YSZ and Ni/GDC pellet sections. Probe/sensor assembly was clamped in a mechanism that translated the sensor across the specimen at a constant speed.

Figure 16. MWM measured permeability vs. bending stress for a low alloy steel specimen made at stresses from $-700 \mathrm{MPa}$ to $+700 \mathrm{MPa}$. Note that in the legend, numbers 1 through 6 for each frequency indicate the sequence of loading.

Figure 17. Experimental setup used to measure stresses in nickel via MWM magnetic permeability measurements. Simplified view of test fixture (top) and actual setup used for measurement.

Figure 18. MWM measured permeability (top) and lift-off (bottom) measured over a range of stresses between $-35 \mathrm{MPa}(-167 \mu$ strain) and $+35 \mathrm{MPa}(+167 \mu$ strain $)$. These values are bending stress and strain at the surface.

Figure 19. Measurements of permeability and lift-off using Mylar shims of various thickness between sensor and material. Measured permeability values vary somewhat with frequency, and are relatively insensitive to differences in lift-off in the 10-25 mil $(250-640 \mu \mathrm{m})$ range, varying by a maximum of $3 \%$ with lift-off across this range.

Figure 20. Scan image of nickel plate (right) and strip (left) after return from laser cutting vendor. Colors represent measured permeability values across surface of plate (refer to scale at bottom of image).

Figure 21. FS35 sensor used in measurement of cobalt-plated brass strip.

Figure 22. Fixture used to measure sensor response to stress changes in cobalt-plated brass. Strip with weight attached to free end was deflected and released so that it oscillated, creating an alternating tension/compression stress condition in the strip.

Figure 23. FS35 sensor response to the stresses created in the oscillating cobalt-plated brass strip. Note that sensor had been calibrated in air, then mounted on the strip to perform this measurement.

Figure 24. Conductivity/Lift-off grid at $25 \mathrm{MHz}$ for a $2 \mathrm{~mm}$ thick cathode. The conductivity range of the grid is $5-20 \mathrm{kS} / \mathrm{m}$. The conductivity of a typical LSM cathode at the operating temperature is $7-15 \mathrm{kS} / \mathrm{m}$.

Figure 25. Thickness/Lift-off grid at $25 \mathrm{MHz}$. The conductivity of the cathode is $10 \mathrm{kS} / \mathrm{m}$. The thickness range for the grid is $0.1-3 \mathrm{~mm}$. 
JENTEK Sensors Inc.

THE ONLY SOLUTION.
110-1 Clematis Avenue, Waltham, MA 02453-7013

Phone: 781-642-9666 - Fax: 781-642-7525

email: jentek@shore.net

Figure 26. Conductivity/Lift-off grid at $25 \mathrm{MHz}$ for a $2 \mathrm{~mm}$ thick cathode. The conductivity range of the grid is $0.5-2 \mathrm{kS} / \mathrm{m}$. The conductivity of a typical LSM cathode at room temperature is $0.5-1 \mathrm{kS} / \mathrm{m}$.

Figure 27. Conductivity/Lift-off grid at $25 \mathrm{MHz}$ for a $50 \mu \mathrm{m}$ (micron) thick cathode. The conductivity range of the grid is $5-20 \mathrm{kS} / \mathrm{m}$.

Figure 28. Thickness/Lift-off grid at $25 \mathrm{MHz}$. The conductivity of the cathode is $10 \mathrm{kS} / \mathrm{m}$. The thickness range for the grid is $5-50 \mu \mathrm{m}$ (microns).

Figure 29. Conductivity/Lift-off grid at $25 \mathrm{MHz}$ for a $50 \mu \mathrm{m}$ (micron) thick cathode. The conductivty range of the grid is $0.5-2 \mathrm{kS} / \mathrm{m}$. The span of this grid is impracticably small, implying that carrying out measurements with it would be extremely difficult or impossible. 


\subsection{EXECUTIVE SUMMARY}

Continued fuel cell development and demonstration projects, bolstered by the recently announced federal funding commitment, are expected to result in affordable, efficient, and clean power sources. A major barrier to their widespread acceptance of Solid Oxide Fuel Cell (SOFC) technology in power generation applications, however, is the high manufacturing and operational cost of SOFC systems. Successful development and implementation of the SOFC technology requires effective nondestructive quality control methods, improved manufacturing process control tools, and SOFC stack health monitoring and control. Improvements in nondestructive tools and methods are expected to reduce life-cycle costs of SOFCs through improved quality, adaptable process control, and timely detection of locally degraded regions within SOFC stacks.

JENTEK has met and exceeded the objectives and task requirements of the Phase I effort. These results indicate that JENTEK's MWM technology can be used to significantly reduce SOFC production and operating costs in a number of ways. The key accomplishments and conclusions from this Phase I study are:

1. Scans of anode material specimens clearly identified regions of high and low porosity. Differences in measured electrical conductivity were found to strongly correlate with differences in porosity, as determined by subsequent metallography. Rapid MWM scans can provide immediate (real-time) nondestructive characterization of porosity for layers that are electrically conductive.

2. MWM measurements demonstrated that stresses in nickel can be detected by measuring changes in its magnetic permeability. Scans of a nickel plate showed that residual stress can be mapped across a surface. Measurements of cobalt-plated brass showed that the stress condition of a nonmagnetizable material, when coated with a thin permeable material, can be monitored. The use of cobalt based coatings may enable monitoring of stresses in the fuel cell e.g., at the IT-SOFC operating conditions.

3. MWM measurements provided rapid, nondestructive, virtually real-time display of results. This capability would enable immediate evaluation of select cell layer properties, instead of the extended time required for other destructive analysis methods, which can often take a week or longer.

4. An investigation of cathode material properties indicated that MWM sensors can be used to monitor select properties such as cathode porosity in cathode supported structures where this layer is relatively thick.

5. An investigation of fuel cell processing methods indicated many opportunities for using MWM and IDED sensors to measure selected properties of electrode and electrolyte layers, such as thickness, conductivity, and dielectric constant.

6. Review of SOFC health monitoring issues, combined with our experience in component health monitoring and prognostics, indicated that high-temperature versions of modelbased sensors have the potential to provide SOFC health monitoring capability. They could also be instrumental in SOFC process control and prognostics. 
JENTEK Sensors Inc.

THE ONLY SOLUTION.

One of the accomplishments in this Phase I program is demonstration of preliminary capability to map porosity in SOFC anode material. This was accomplished in blind tests without a priori knowledge of the porosity distribution and without calibration standards. This unique capability to perform property measurements without calibration standards, i.e., using calibration in air, is described in a recently issued ASTM Standard E2338-04 "Standard Practice for Characterization of Coatings Using Conformable Eddy Current Sensors without Reference Standards". The porosity maps generated by the Meandering Winding Magnetometers MWM $^{\circledR}$-Array were subsequently confirmed by metallography. This is just one example of the advanced capabilities that will be enabled by the application of JENTEK technology to characterization of fuel cell components. 


\subsection{BACKGROUND}

JENTEK has performed a thorough review of state-of-the-art practices and characterization methods for SOFCs. This section provides a brief summary of this effort and our assessment of the technology commercialization opportunities.

\subsection{Current Manufacturing Practice for SOFCs}

The most successful state-of-the-art high-temperature Solid Oxide Fuel Cells are commercially available from Siemens Westinghouse Power Corporation. They operate at around $1000^{\circ} \mathrm{C}$, with fuel utilization of $80-90 \%$, and power densities in the range of $0.2-0.5 \mathrm{~W} / \mathrm{cm}^{2}$ [Siemens, 2004]. The anode, electrolyte, cathode and interconnect materials are Ni-yttria stabilized zirconia (YSZ) cermet (electronic conductor), YSZ (oxygen-ion conductor), A-site (Sr) doped lanthanum manganite (electronic conductor), and doped lanthanum chromite (electronic conductor), respectively. In the Siemens Westinghouse SOFC, the electrodes (anode and cathode) are 30$40 \%$ porous and permit molecular diffusion of gases, and the electrolyte and interconnect are dense. The cathode (1-2 mm thick) is fabricated by green extrusion followed by sintering at elevated temperature. Until very recently the electrolyte (20-40 $\mu \mathrm{m}$ thick) was deposited through the electrochemical vapor deposition (EVD) process; however, Siemens Westinghouse is currently transitioning to a thermal spray process for electrolyte deposition. The anode (100-150 $\mu \mathrm{m}$ thick) was until very recently deposited by slurry coating followed by EVD fixing; however, Siemens is currently transitioning to a thermal spray process for the anode layer as well. The interconnect (50-100 $\mu \mathrm{m}$ thick) which covers a small fraction of the circumference of the cathode is presently deposited using a plasma-spray process.

Fabrication of anode supported planar fuel cells proceeds through the following steps. First $\mathrm{NiO}-\mathrm{YSZ}$ tapes are fabricated using a standard tape-casting process. YSZ is then deposited either by spray coating the NiO-YSZ tapes using an air gun or cast using a second tape-casting process and laminated to the anode tape using a tape calendaring process. The NiO-YSZ/YSZ bi-layers are then fired at elevated temperature $\left(1300-1400^{\circ} \mathrm{C}\right)$ to densify the YSZ electrolyte layer and to sinter-bond the NiO-YSZ anode to the electrolyte. The next step fabrication is deposition of the cathode using a spray-coating or screen-printing technique followed by a second firing step at a lower temperature $\left(\sim 1000-1100^{\circ} \mathrm{C}\right)$ to sinter-bond a porous cathode film to the electrolyte. Single cells formed in this manner are then placed between bi-polar interconnect plates and bonded to them using an appropriate joining method (this is still a very active area of research and development). The NiO-YSZ anode is usually reduced to Ni-YSZ during operation of the cell/stack.

\subsection{State-of-the-art in SOFC characterization}

State-of-the-art characterization techniques for SOFCs fall under two broad categories: microstructural characterization and electrochemical characterization. 


\subsubsection{Microstructural characterization}

Microstructural characterization of SOFCs is usually performed as part of the quality control process and also as part of post electrochemical test characterization. Characterization typically involves resin-mounting sections of SOFCs or SOFC components using appropriate metallographic techniques followed by polishing and scanning electron microscopy (SEM). Electrode porosity is usually measured using the line intercept technique, i.e., by drawing several lines on the micrograph of the polished sections at equal angular intervals and measuring the fraction of the line inside pore space to the total length of the line. Other techniques of measuring pore volume fraction from polished cross sections of electrodes include using quantitative metallography techniques with macros that can be set up in software packages like Adobe Photoshop. Unfortunately, all current microstructural characterization techniques are destructive.

Current techniques are also slow. Presently, microstructural characterization takes from seven to ten days to complete. Microstructural characterization does provide comprehensive information on many features of the cell including component thicknesses, electrode porosity, adherence of components and the state of the electrode-electrolyte interfaces. In addition, polished cross sections of the cell can be used for chemical analysis of the electrode and electrolyte using energy and/or wavelength dispersive spectroscopic analysis, scanning electron microscopy and electron microprobe analysis (SEM-EDX and EPMA). In many instances, however, using current microstructural characterization techniques for the limited goal of obtaining electrode porosity and porosity distribution across the electrode thickness requires a disproportionate investment in time and labor. It is here that JENTEK's model-based sensor technologies offer a powerful alternative. These sensors can promptly characterize microstructural variations, and layer thickness. This nondestructive characterization can be used at different stages of SOFC fabrication, testing, and operation. Furthermore, real-time porosity measurement enables realtime modification of process parameters and even real time control.

\subsubsection{Permeability and diffusivity characterization.}

Another technique used to characterize porous SOFC electrodes is the measurement of gas permeability and diffusivity. Permeability and diffusivity are parameters that characterize the flow of gases through porous media via viscous transport and diffusion respectively.

During a typical gas permeability measurement, the porous electrode is exposed to gases with identical composition (i.e., no concentration gradient) and the flow rate of the gas through the pores is measured under a fixed pressure gradient. When the concentration of gases is uniform on both sides, the only mechanism by which transport of gases can occur through the pores is viscous transport, i.e., convective flow. Thus, permeability is a measure of the contribution of viscous transport mechanisms to gas flow in the pores.

By contrast, diffusivity measurements are made through a setup such as a Graham diffusion cell. In such a measurement, the transport of gases through pores due to a concentration gradient alone is measured. Thus, diffusivity is a measure of the contribution of diffusive transport to gas flow in the pores. 
In a typical cell of practical dimensions (e.g., a plate with a $10 \mathrm{~cm} \times 10 \mathrm{~cm}$ area) or a $2 \mathrm{~m}$ long tube as in the case of the Siemens Westinghouse cathode substrate, the porosity distribution is expected to vary either intentionally, by design, or unintentionally, due to poor quality control during the manufacturing process. While both of the above mentioned parameters, i.e., diffusivity and permeability, are important in describing gas transport through pores in the electrode and give indirect information about electrode porosity, such measurements are conducted over selected sections of the cell. Thus, to perform such measurements, one would be forced to section the cell into pieces of appropriate dimensions. Therefore, measurements of permeability and diffusivity of the electrode are destructive techniques as well. Also, current techniques can not provide a complete image of porosity variations across the surface.

While permeability and diffusivity measurements are valuable characterization tools that are especially helpful during process development for SOFC components, their destructive and timeconsuming nature make them considerably less than ideal as quality control tools in a manufacturing environment. Again, the nondestructive characterization of electrode porosity with JENTEK's model-based sensors can provide significant advantages, including real-time measurements, high resolution imaging of the entire surface and reliable quantitative measurements.

Based on our successes in characterization of high-temperature coatings under a complementary Department of Energy Phase II SBIR (titled "Intelligent Probes for Enhanced Nondestructive Determination of Degradation in Hot-Gas-Path Components"), it is expected that, in addition to microstructure/porosity characterization demonstrated in this Phase I, layer thickness measurement/mapping for SOFCs should also be possible. The independent measurements of porosity and thickness represent unique and patented capabilities of JENTEK sensors. Combination of these capabilities would result in significant cost savings by reducing the percentage of defective SOFCs.

\subsection{Reducing SOFC Cost}

As described on the National Energy Technology Laboratory (NETL) website (http://www.seca.doe.gov/), "The Solid State Energy Conversion Alliance's (SECA) goal is to create a solid oxide fuel cell (3-10 KW) that can be mass-produced in modular form at a target cost of $\$ 400 / \mathrm{kW}$." Clearly, much must be accomplished to achieve this goal, and one area that is critical to achieving success is tight quality control in the fabrication of electrode/electrolyte assemblies (EEAs). This point is emphasized in Figure 1, taken from a DOE cost model study of SOFC technology performed by TIAX [Carlson, 2004]. As shown by this cost model, "assembled stack cost will be highly sensitive to the percentage of defective EEA's. For example, for the manufacturing process, a $1 \%$ defect level could increase the stack cost from $\$ 92 / \mathrm{kW}$ to $\$ 278 / \mathrm{kW}$.

JENTEK's model-based sensors can be used to address this cost challenge by enhancing quality control capability. The rapid, nondestructive measurements enabled by these sensors are expected to allow immediate measurement of key material properties in the EEAs and in the 
JENTEK Sensors Inc.

THE ONLY SOLUTION.
110-1 Clematis Avenue, Waltham, MA 02453-7013

Phone: 781-642-9666 - Fax: 781-642-7525

email: jentek@shore.net

electrode/electrolyte layers during their manufacture. With this enhanced quality control capability, defective EEAs can be more effectively identified and removed before being assembled into the stack.

Another important result from the TIAX cost model study is the sensitivity of EEA cost $(\$ / \mathrm{kW})$ to cell power density, as shown in Figure 2 [Carlson, 2004]. Again, the effect is dramatic. As reported in this study, "Achieving high power densities is critical to lower stack cost." JENTEK's model-based sensors can be used to maximize cell power density by assuring uniformity of material across the functional area of the fuel cell. By mapping key material properties across the surface during fabrication of the cell layers, measures can be taken to reduce variability across the cell surface, improving the likelihood that the cell performs to specification across its full surface.

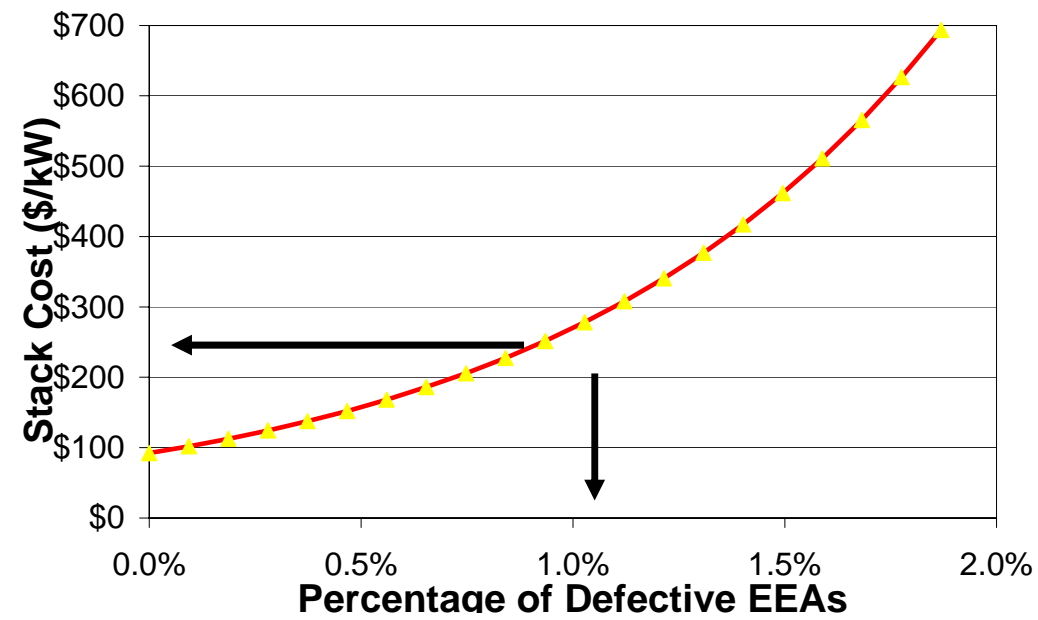

Figure 1. SOFC stack cost vs. percentage of defective Electrode/Electrolyte Assemblies (EEAs) [Carlson, 2004]. 
JENTEK Sensors Inc.

THE ONLY SOLUTION.

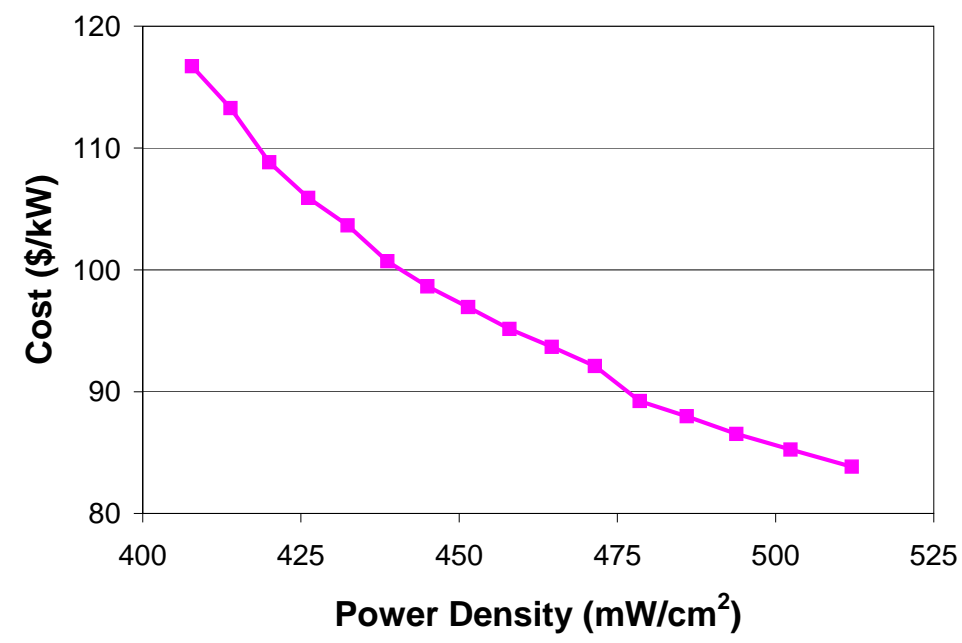

\begin{tabular}{|l|c|c|}
\cline { 3 - 3 } \multicolumn{1}{c|}{} & $\begin{array}{c}\text { Layer } \\
\text { Thickness } \\
(\mu \mathrm{m})\end{array}$ & Status \\
\cline { 3 - 3 } \multicolumn{1}{c|}{ Anode } & $\mathbf{7 0 0}$ & Fixed \\
\hline Cathode & $\mathbf{5 0}$ & Fixed \\
\hline Electrolyte & $\mathbf{5 ~ 2 0}$ & Vary \\
\hline
\end{tabular}

Figure 2. SOFC stack cost vs. power density [Carlson, 2004].

\subsection{Health Monitoring of SOFCs}

In the area of SOFC health monitoring, model-based sensors are likely to provide early detection of locally degraded regions. This could be accomplished either during shutdowns, using scanning sensors, or on-line, using permanently mounted high-temperature sensors. In both cases, the sensors will monitor material degradation. In the case of the high-temperature sensors, however, SOFC operating parameters and/or process dynamics will also be monitored. Both modes of SOFC health monitoring should allow for timely detection of failing fuel cell elements and identification of the root cause(s) of failures. The key is to monitor all relevant usage, damage and precursor states. For fuel cells, usage states include temperature and stress, while damage states may include microstructural changes, cracking, and local corrosion, and precursor states may include initial microstructural/porosity variations and residual stresses.

State-of-the-art SOFC field demonstration systems are highly instrumented and designed to obtain system level operational information such as stack and row voltages and currents, temperature, air and fuel gas composition and flow rates (which is directly related to the air and fuel utilization), system pressure and many other parameters. The overall health of such systems during operation is monitored mainly by combining information regarding stack and row voltages, currents and stack temperatures from various locations within the stack. While this methodology is very successful at obtaining a real-time health report of the SOFC power system, it provides little information on the health of individual cells. For instance, there is presently no easy way to obtain answers to questions such as:

- What is the initial porosity distribution of the electrodes in individual cells in the stack as a function of position in the stack? How does it change with time?

- What is the initial stress distribution between the various cell component layers and how does it change with time and position within the stack? 
- How does the stress distribution change during involuntary or voluntary thermal cycling of the stack?

- What are the temperatures at buried interfaces within a cell during operation?

The combination of the high operating temperature and current density could have a profound effect on electrode microstructure and porosity. Changing porosity distribution or microstructure within the electrodes could shift the current density distribution in the cells which in turn could alter the temperature distribution in the stack. In fact, microstructural analysis of cells after long-term operation often reveals significant microstructural and porosity distribution changes particularly at the interfaces between the electrodes and electrolyte of the cell.

In particular, long-term operation results in densification of the cathode and reduction in porosity at the cathode-electrolyte interface. It is likely that such densification is worse at higher current densities due to changing point defect chemistry of the cathode material at high current densities. Reduction in porosity would most certainly be accompanied by an increase in one type of polarization loss in the cell known as concentration polarization. Concentration polarization is the voltage loss that occurs in the electrodes due to resistance to gas transport. In an extreme scenario, the sequence of changing porosity engendered by temperature distribution leading to shifting current density distribution, which in turn leads to a different temperature distribution, could set up an undesirable runaway positive feedback loop. This could occur over a long period of time with a long time lag between the occurrence of underlying porosity and stress changes and consequent negative effect on the performance of the system.

Thus, a tool which can monitor changes in porosity distribution, stress, and temperature at individual cells in the stack (and near interfaces within those cells) in real time would provide a substantial benefit. Such a tool would provide information which can be used not only to take corrective action during operation but also in the design of SOFC cell and stack components.

In this Phase I and in related programs, JENTEK's MWM and Interdigitative Electrode Dielectrometer IDED ${ }^{\circledR}$ technology have already demonstrated key capabilities needed to realize such an SOFC monitoring capability. This has included (1) stress measurement for nickel; (2) use of cobalt coatings to enhance stress measurement sensitivity, suitable for buried interfaces; (3) through layer temperature measurement, suitable for monitoring temperature at buried interfaces; and (4) porosity and microstructure characterization for electrodes. All of these measurements are made nondestructively and in real time. In future work, these capabilities can be extended through tasks focused on specific SOFC requirements for health monitoring.

Capabilities of the inductive model-based sensors, e.g., MWMs and MWM-Arrays, include measurements of electrical conductivity, magnetic permeability, lift-off, and layer thickness. The dielectrometers, such as the interdigitated electrode dielectrometers (IDEDs), offer the capability to measure dielectric properties, lift-off, and layer thicknesses of nonconductive materials. The dielectric properties include dielectric constant (permittivity) and electrical conductivity (for slightly conducting or lossy dielectrics). 
The ultimate goal is to effect significant life-cycle cost savings. This goal can be achieved through implementation of model-based sensors in combination with powerful multivariate methods demonstrated successfully in our DOE Phase II SBIR effort for land-based turbine components. These methods empower specific SOFC-relevant capabilities of the model-based sensors to provide numerous capabilities, including porosity mapping, material condition/degradation monitoring, stress monitoring (for ferromagnetic materials), temperature monitoring, and surface roughness estimation.

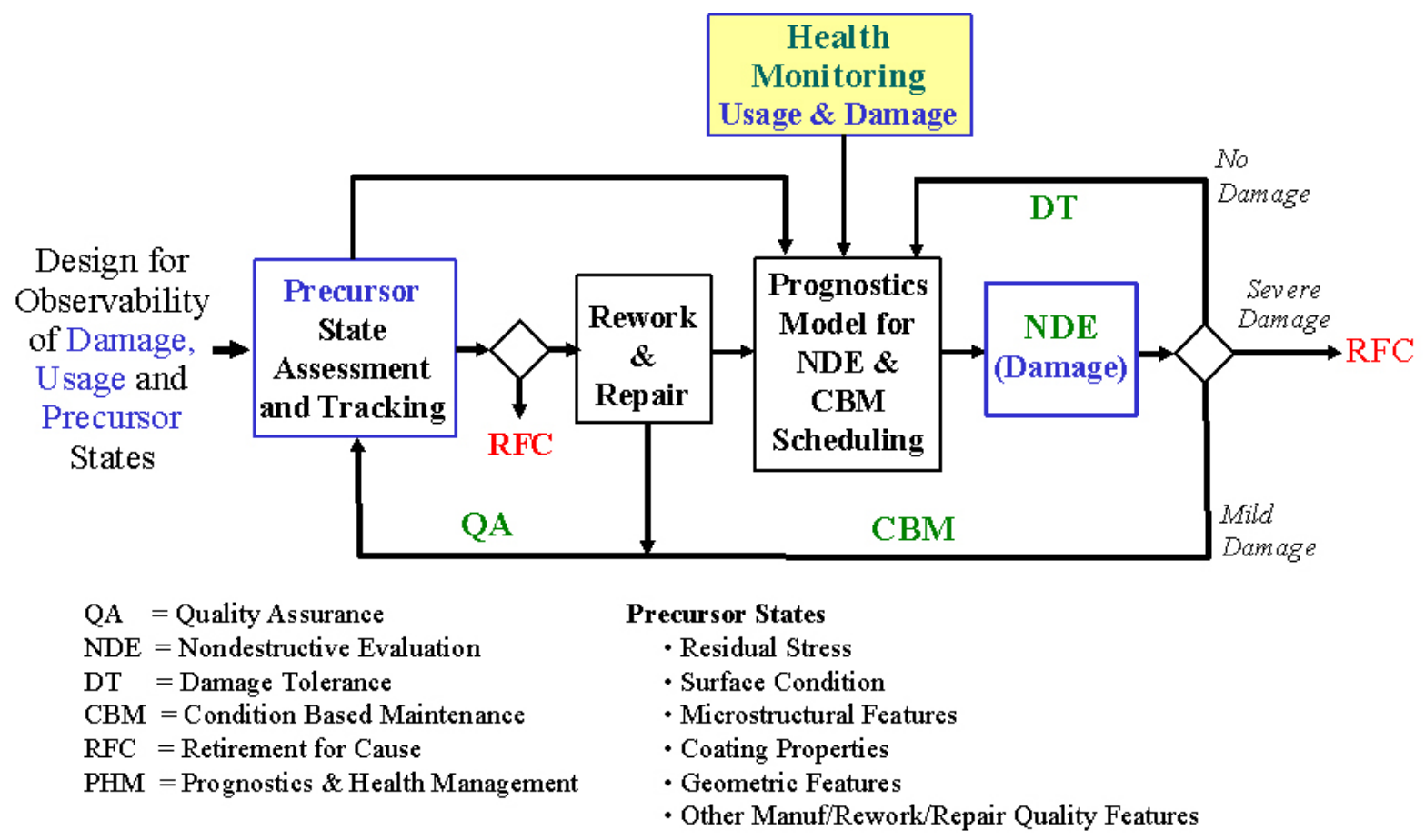

Figure 3. Adaptive damage tolerance flow chart.

Figure 3 illustrates a health monitoring framework that is being developed by JENTEK for aerospace applications called Adaptive Damage Tolerance (ADT). It is expected that this framework can be applied to the QA, NDE, CBM and PHM for fuel cell life-cycle management.

\subsection{Commercialization Opportunity}

The fuel cell power generation market is poised to grow exponentially over the next decade. In fact, according to a recent SOFC market study, "the fuel cell industry is one of the fastest growing industry segments of the power industry" [Colson-Inam, 2004]. Early signs of this trend are (1) sustained expansion of fuel cell development and demonstration projects with a major focus on dramatic cost reduction; (2) positioning of all major power generation OEMs for the expected expansion of the fuel cell market; and (3) government initiatives to hasten both fuel cell R \& D and transitioning of successes to the marketplace. Power systems divisions of OEMs, like Siemens Westinghouse, GE Power Systems, Mitsubishi, Rolls Royce and a number of other power generation OEMs are actively pursuing efforts leading to commercialization of current SOFCs, and considerable future expansion of SOFCs in power generation. 
Early development and introduction of effective quality assessment, process control, and health monitoring tools are crucial to reduction of total ownership cost for future SOFC plants. As in traditional power generation, the SOFC plant OEMs will be looking for new technologies to offset their anticipated warranty costs on delivered SOFC units. The need to avoid down time and minimize repair/replacement costs will be driving expansion of the market for sensor technologies that will be instrumental in achieving these goals.

Thus, JENTEK's focused effort on development of our sensor technologies for SOFC stack health monitoring and process control, as well as for manufacturing process control and quality assessment, is well timed. Our current product line offers relatively low cost tools that have shown significant value in a number of power plant applications. JENTEK believes it can support the growth of the SOFC industry by providing the following new capabilities:

1. A means of effective quality assessment for SOFC components,

2. Manufacturing process control tools aimed at improvement of SOFC component quality and reduction of manufacturing costs, and

3. Condition monitoring capability, through both permanently mounted and scanning sensor configurations that will permit prognostics for and health management of the SOFC stack with the objective of reducing total ownership costs.

In this Phase I SBIR effort, JENTEK demonstrated feasibility of porosity mapping for SOFC electrodes and stress measurement in nickel and cobalt. We also have identified a number of potential applications of model-based sensors and have started a dialogue with SOFC developers including a major SOFC stack/SOFC plant OEM, as well as with Department of Defense organizations. These and similar future efforts will significantly reduce inherent barriers to broad commercial acceptance and use of our products and technology in this emerging energy sector.

JENTEK has filed a provisional patent application which is based on SOFC related developments from JENTEK IR\&D. We expect to continue our presence at the fuel cell conferences and to file numerous additional patents that will substantially enhance our competitive advantage and ensure successful commercialization and expanding market share for our products and services.

This technology has potential to directly benefit (a) OEMs, who will be facing substantial warranty rework for delivered SOFC units; (b) operators, who cannot afford unplanned service interruptions and must offer reliable power to their customers; (c) developers of next generation SOFC systems who need more reliable tools for anticipating total ownership costs and who are moving on to a "design for life management" philosophy that is dynamic and progressive; and (d) the federal government that is ultimately responsible for mitigating risks of power interruption and excessive costs to businesses and individuals. 


\subsection{RESULTS OF PHASE I AND RELATED PROGRAMS}

\subsection{Summary of Phase I Accomplishments}

The Phase I effort proved to be very successful. The Phase I objectives have each been completely satisfied and exceeded by the Phase I research. This section describes the Phase I results that demonstrate the feasibility of the MWM technology as well as already demonstrated capability. After a brief background section, specific results are provided that meet the stated Phase I objectives.

The Phase I objectives (abbreviated here) were:

Objective 1: Establish the capability and limitations of current model-based sensors for characterization of solid oxide fuel cell materials.

Objective 2: Demonstrate MWM sensor or MWM-Array sensitivity to porosity measurements in SOFC electrodes and establish potential improvements with planned Phase II enhancements.

Objective 3: Investigate the potential of MWM sensors to measure stresses in the anode metal matrix at ambient temperature.

Objective 4: Investigate the potential of model-based sensors to provide characterization of other properties of SOFC materials.

Objective 5: Investigate the potential of model-based sensors to provide condition monitoring of SOFC materials.

Objective 6: Investigate scale-up issues for model-based sensors that indicate the potential as a materials characterization tool for future production of SOFCs.

The Phase I Work Plan tasks were:

Task 1: Acquire relevant specimens.

Task 2: Adapt MWM-Array sensors and instrumentation for porosity characterization in the anode metal matrix material at ambient temperature.

Task 3: Adapt MWM-Array sensors for stress measurements in the anode metal matrix material near the anode/electrolyte interface at ambient temperature.

Task 4: Investigate the potential for using a high-temperature version of an MWM-sensor or MWM-Array to characterize cathode properties at high temperatures.

Task 5: Identify another property of interest for a relevant fuel cell component and investigate measurement feasibility.

Task 6: Investigate the potential for model-based sensors to provide monitoring and control of SOFC components using inductive and/or dielectric sensors.

Task 7: Investigate scale-up issues and provide recommendations for Phase II enhancements.

Task 8: Phase I final report.

JENTEK has met and exceeded the objectives and task requirements of the Phase I effort. In this Phase I SBIR, JENTEK has identified ways in which its model-based sensors can be used to 
reduce costs. JENTEK's sensors can achieve this through enhanced quality assessment and process control for critical stack components. Demonstrated and anticipated capabilities to reduce SOFC manufacturing and operational cost include:

- Electrode porosity mapping/monitoring,

- Layer thickness measurements/monitoring,

- Electrode and metallic interconnect conductivity monitoring, e.g., for degradation assessment,

- Stress measurements in anodes and some interconnect materials (even through layers),

- Surface roughness measurements for metallic and cermet components,

- Temperature measurement through layers and at interfaces, and

- Rapid nondestructive measurements without calibration standards.

This section first describes the model-based sensors and measurement grid methods. Some of these sensors have been used in Phase I, while others are currently under development. This is followed by detailed results of the work performed under Phase I.

\subsection{Model-based Sensors, Description of Technology}

\subsubsection{The Meandering Winding Magnetometer (MWM)}

The conformable MWM eddy current sensors and MWM-Arrays provide advanced inspection capabilities for complex geometry components. The sensors, which operate in the magnetoquasistatic (MQS) regime, provide measurements and mapping of absolute electrical conductivity and magnetic permeability, which can be correlated with many material conditions of interest. Layer thickness variations can also be measured with high resolution. The sensors can be operated over a wide range of frequencies $(100 \mathrm{~Hz}$ to $31.6 \mathrm{MHz})$ and can provide bidirectional measurements to permit optimization of sensitivity to a particular condition. Changes in conductivity or magnetic permeability revealed by multi-frequency multi-directional measurements using conformable MWMs and MWM-Arrays have been correlated with many conditions of interest for production quality control and component life management, including characterization of MCrAlY and PtAl coatings on turbine blades.

Examples of MWMs and MWM-Arrays are shown in Figure 4. The original MWM sensor (Figure 4a) consisted of a meandering primary winding with secondary windings placed on either side of the longer segments of the primary. Figure $4 \mathrm{~b}$ shows an enhanced MWM construct that can be modeled more accurately from basic physical principles using rapid quasi-analytical methods. Driving the primary winding with an alternating current creates a magnetic field that interacts with the material under test. For conducting materials, the induced eddy current pattern follows the winding pattern of the primary. A single element sensor has multiple secondary windings, i.e., sensing elements connected together so that a single output voltage is measured. The absolute impedance (sensing element voltage/drive winding current) is measured using a JENTEK impedance instrument. In an MWM-Array, multiple relatively small sensing elements, 
JENTEK'Sensors Inc.

THE ONLY SOLUTION.
110-1 Clematis Avenue, Waltham, MA 02453-7013

Phone: 781-642-9666 - Fax: 781-642-7525

email: jentek@shore.net

e.g., $1 \mathrm{~mm}$ by $1 \mathrm{~mm}$ square, are located between longer segments of a single rectangular shape drive winding (see Figure 4c). The parallel architecture impedance instrument measures the impedance for each sensing element. In a scanning mode, these sensing elements permit highresolution imaging of material properties associated with thermal damage and imaging of discontinuities such as cracks. In a segmented field MWM-Array (Figure 5d), rows of sensing elements are located at different distances from a center drive winding to enable multiple depths of sensitivity at each applied input current frequency. This will be valuable when assessing properties, e.g., porosity, that may produce dispersive (i.e., frequency dependent) "effective" electrical conductivity or magnetic permeability.

The MWM and MWM-Array sensors are fabricated on a thin and flexible substrate using microfabrication techniques, producing essentially identical conformable sensors with essentially identical performance. The repeatability of these sensors provides an important advantage over coil type eddy current sensors, for which nominally identical probes (terminal inductances within $2 \%$ ) have been found to produce signals that differ by as much as 35\% [Auld, 1999].

The windings of the MWM sensors (Figure 4), resembling a square wave, are designed to produce a spatially periodic field. This field shape permits rapid and accurate modeling of the interaction with the material under test, thus significantly reducing calibration requirements. For example, in some situations an "air calibration" can be used, permitting measurement of a component's absolute electrical conductivity without calibration standards. For characterization of coatings, for example, calibration can be performed on an uncoated reference part with the approximate conductivity of the substrate (such as an uncoated section of material) as described in ASTM standard E2338-04 [ASTM, 2004]. 


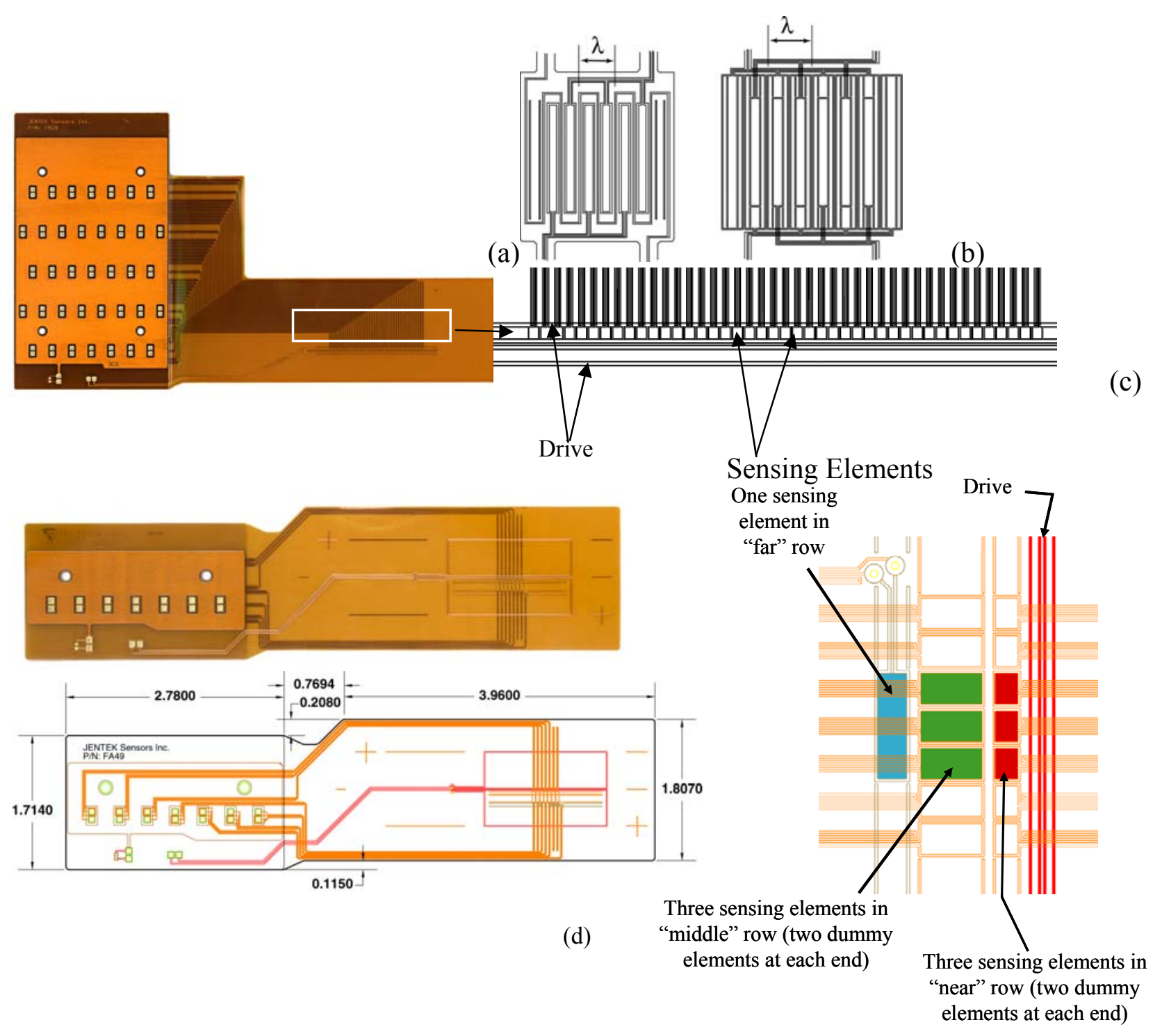

Figure 4. (a) Original MWM sensor construct, (b) enhanced MWM design, (c) example MWM-Array, (d) example segmented field MWM-Array.

The winding pattern and resulting field shape also result in a strongly directional sensor. This directionality can provide a powerful diagnostic tool. For example, the sensor is strongly sensitive to stress changes in magnetizable materials and to cracks oriented perpendicular to the longer segments of the winding. This latter MWM capability permits determination of crack orientation. On the other hand, round pits or voids produce an equal response in either sensor orientation. Thus, the ratio of responses can provide information about the nature of the flaw. Bidirectional MWM measurements are used to (1) characterize material anisotropy, (2) estimate stresses in magnetizable materials, including nickel, cobalt, iron, many of their alloys, and steels, 
and (3) obtain other valuable information for various material condition characterization and component inspection tasks.

Scanning with MWM sensors or with MWM-Arrays provides the capability for imaging material properties and conditions, and, thus, significantly simplifies interpretation of inspection results. The related work section provides several examples of MWM and MWM-Array results in addition to those provided in the remainder of this section.

\subsubsection{Interdigitated Electrode Dielectrometer (IDED)}

The basic structure of the JENTEK capacitive sensor is the IDED shown in Figure 5a. A JENTEK IDED sensor, which operates in the electroquasistatic mode, and enclosure are shown in Figure 5b. One set of electrode fingers are driven by a sinusoidally time varying signal with known amplitude and the second set of interdigitated fingers are virtually grounded and the terminal current constitutes the sensed signal.

For layered media or materials having dielectric properties that vary with depth, the measured transadmittance (sensing electrode terminal current divided by the driven electrode voltage) between the drive and sense electrode, which measures the effective complex permittivity of the material, is a thickness-and depth-weighted response of the dielectric properties of the various regions. Typical excitation frequencies range from $0.005 \mathrm{~Hz}$ for highly insulating materials to 10 $\mathrm{MHz}$ for semiconducting materials.

The depth of penetration of the electric field into the material is proportional to the spatial wavelength of the periodic electrodes. The periodic variation of electric potential along the surface in the $x$ direction (see Figure 5a) produces an exponentially decaying electric field that penetrates into the medium in the $z$ direction. The depth of penetration of the electric field is approximately $1 / 3$ of the spatial period. However, the depth of sensitivity will depend on the noise levels and the required level of sensitivity for a specific property or feature of interest. This implies that small wavelength sensors will primarily respond to changes of material properties near the sensor-material interface, while larger wavelength sensors respond to changes farther from the sensor interface. Thus, multiple wavelength sensors can be used to measure spatial profiles of dielectric properties [Melcher, US Patent 4,814,690].

Several types of multiple wavelength sensors have been developed by JENTEK. In one approach, multiple sets of interdigitated spatially periodic electrodes are laid out on a common substrate, as shown in Figure 6a. While this provides distinct wavelength IDEDs, each IDED is sensitive to different regions of the test material. As a result, if there is a spatial variation in the thickness or dielectric properties of the test material, or if there is an air gap variation, for example, due to uneven pressure against the material or dust particles, it can be difficult to combine the measurements for meaningful property estimates. 
JENTEK Sensors Inc.

THE ONLY SOLUTION.

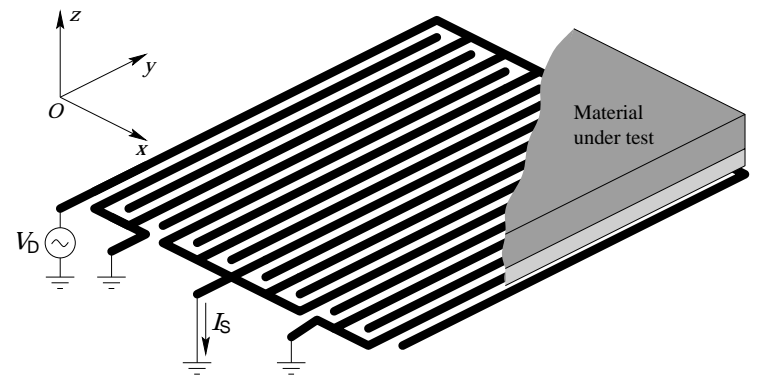

(a)

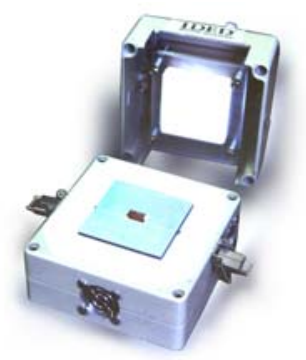

(b)

Figure 5. (a) Interdigitated electrode (IDED) sensor in contact with a test material; and (b) commercially available JENTEK sensor and enclosure for capacitive sensing of dielectric samples.

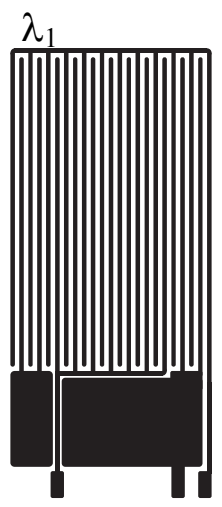

(a)

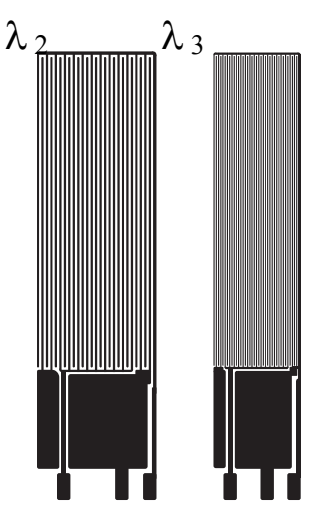

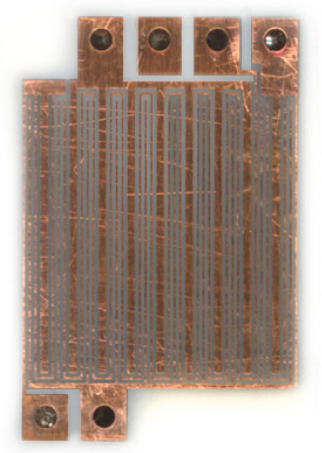

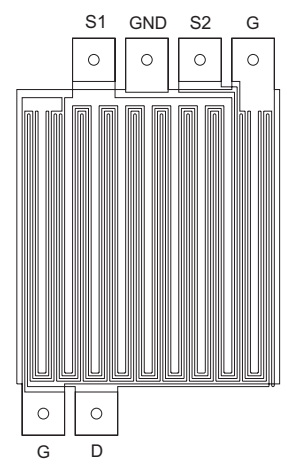

(b)

Figure 6. (a) A three-wavelength sensor with three separate sensing regions of different wavelength formed on a Teflon substrate with wavelengths of $5 \mathrm{~mm}, 2.5 \mathrm{~mm}$, and $1 \mathrm{~mm}$. (b) Photograph and corresponding schematic for a two-wavelength co-located interdigitated electrode sensor.

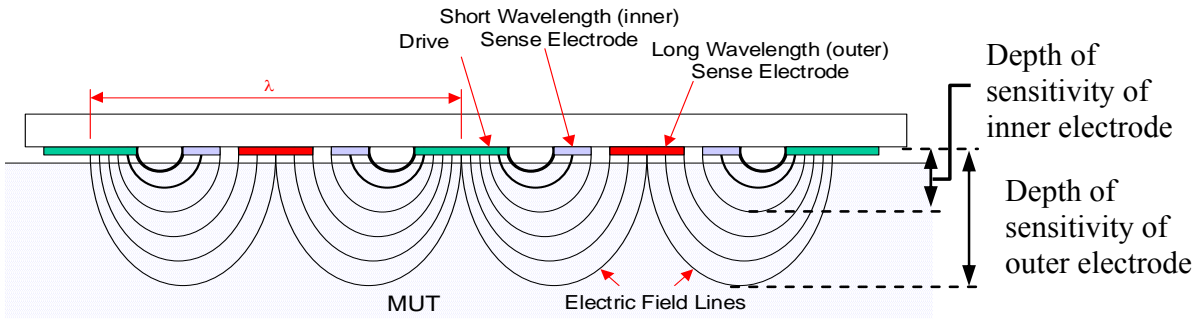

Figure 7. Multiple co-located dielectric sensors sense the same region of the test material, ensuring that the longer and shorter wavelengths have the same average lift-off or air gap thickness.

The solution, also developed and patented by JENTEK, is to integrate multiple sensing elements into a single sensing structure so that all of the sensing elements interrogate the same region of 
the material under test, as illustrated in Figure 6b [Schlicker, 2002]. A schematic for the electric field distribution is shown in Figure 7, where multiple sensing electrodes are placed within each interdigitated electrode period and respond to different effective wavelength (short or long) modes of the electric field. Thus, for this sensor, the lift-off and dielectric constant can be measured independently. Figure 8 shows an alternative cylindrical version of this approach demonstrated by Shay [2001], and Schlicker [2002].

Sensing Electrodes
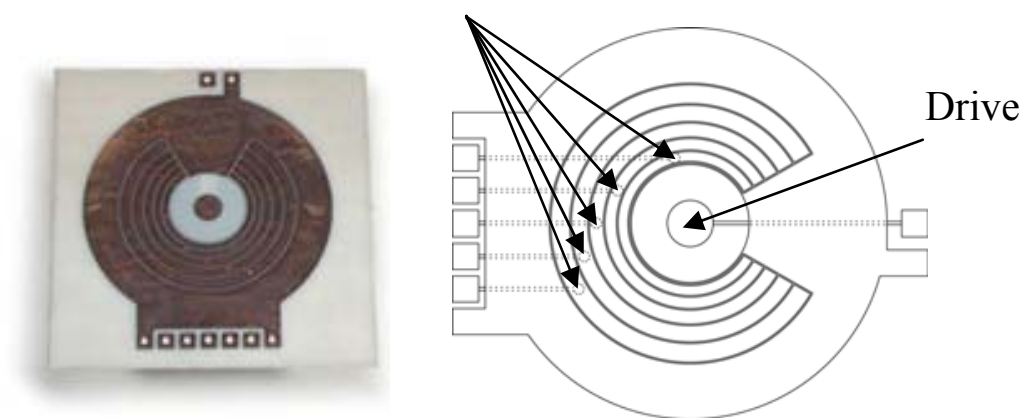

Figure 8. Segmented-field IDED sensor with rotational symmetry.

Recently, JENTEK developed a flexible IDED sensor that can be used for characterization of complex geometry components, including cylindrical layers in a tubular SOFC.

\subsubsection{Measurement Grid-Based Inversion Methods}

\section{Grid-based Inversion Methods}

The MWM, MWM-Array or IDED sensing element response is converted into material or geometric properties using measurement grids. These grids are used to map the magnitude and phase of the transimpedance or transadmittance into unknown properties of interest. Background for the measurement grid-based inversion methods was originally described by Goldfine [1990, 1993]. The grids are two-dimensional pre-computed databases of responses that are used to calibrate and perform measurements of absolute material properties. Typically, grids relate two measured parameters to two unknowns, such as the electrical conductivity and lift-off, or metallic coating thickness and lift-off, or dielectric constant and lift-off as shown in Figures 9 and 10. Figure 11 shows a flow chart of the grid generation process. For solution of three unknown problems, three-dimensional versions called lattices are used, and for four (or more) unknown problems, these sensor response databases are called hypercubes. 
JENTEK Sensors Inc.

THE ONLY SOLUTION.
110-1 Clematis Avenue, Waltham, MA 02453-7013

Phone: 781-642-9666 • Fax: 781-642-7525

email: jentek@shore.net

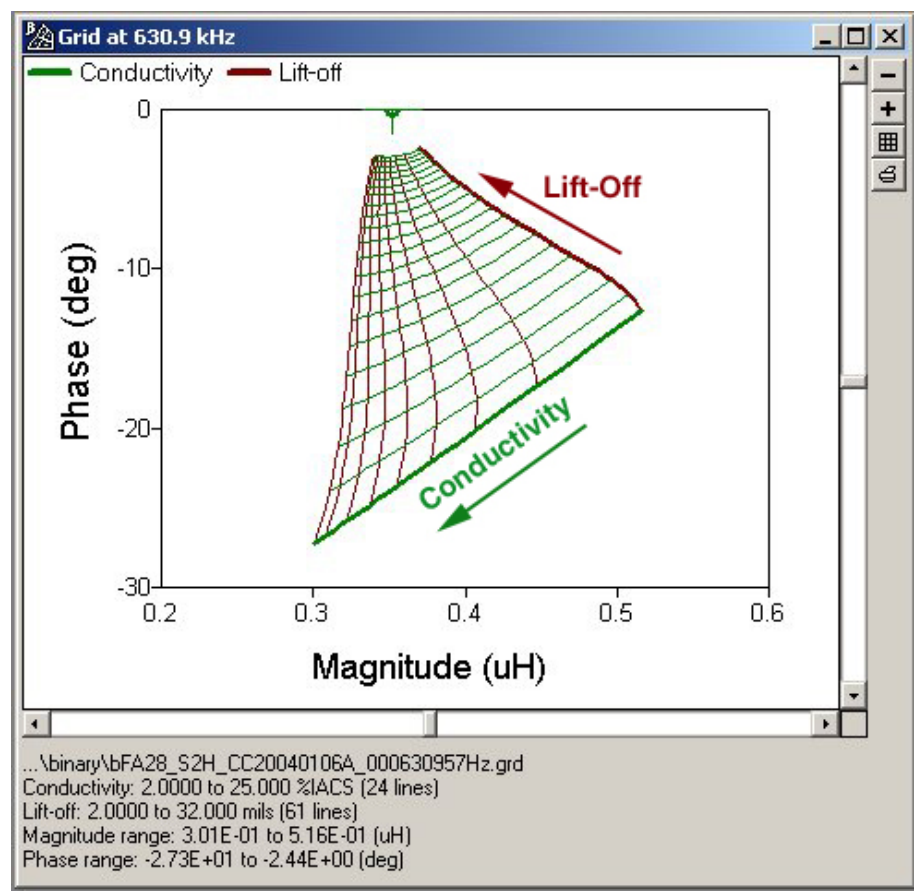

Figure 9. $630 \mathrm{kHz}$ conductivity/lift-off grid generated for conductivities of 2-25\%IACS and lift off values of 2-32 mils. Grids are used to rapidly convert sensor response into material property values.

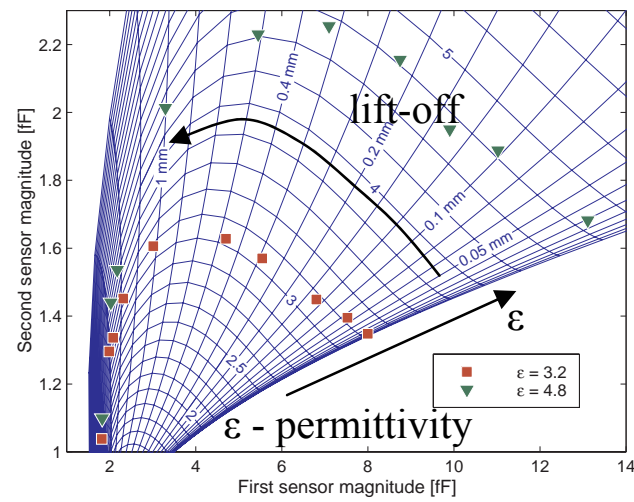

Figure 10. Dielectric constant/lift-off measurement grid and results of two-unknown measurements carried out with a circular IDED. 


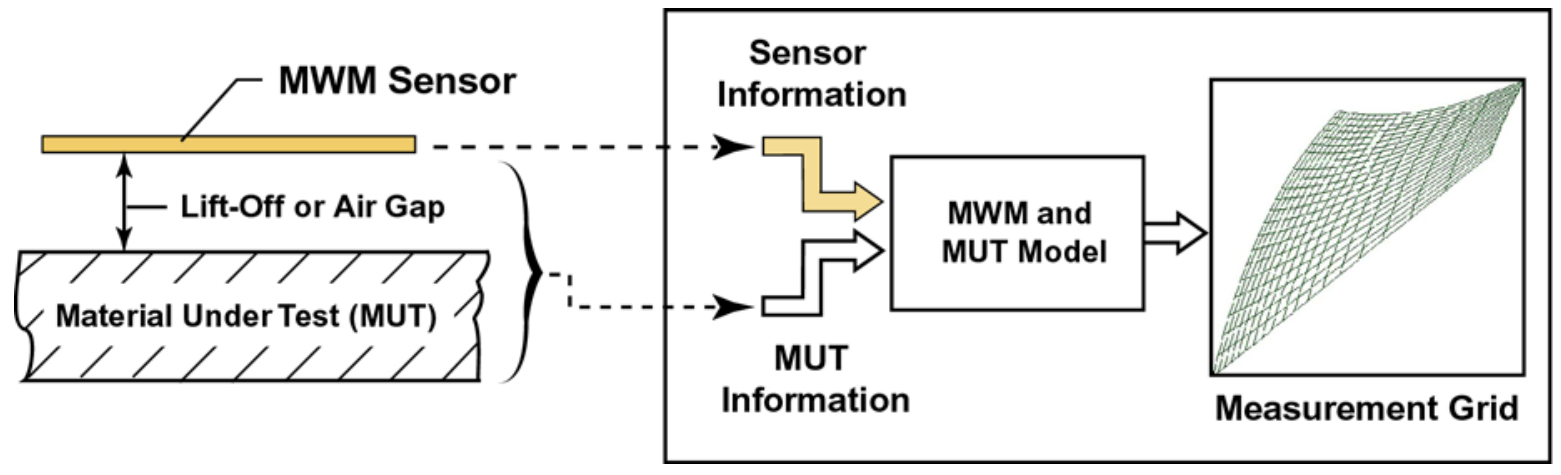

Figure 11. Flow chart of grid (sensor response database) generation process.

\subsection{MWM-Array Porosity Mapping}

This section summarizes results of Tasks 1 \& 2, i.e., acquisition of relevant specimens, and adaptation of MWM-Array sensors and instrumentation for porosity characterization in the anode cermet material at ambient temperature.

\subsubsection{Tasks 1 and 2 overview}

Pellets representative of SOFC anodes were prepared by Prof. Srikanth Gopalan of Boston University. These pellets were nondestructively scanned by JENTEK using an MWM-Array sensor to produce electrical conductivity images, then sectioned metallographically at Boston University to compare MWM measurements to porosity levels in the pellets. A high correlation was found between the MWM-Array measurements of conductivity and metallographic determination of porosity.

\subsubsection{Pellet fabrication procedure}

Pellet specimens of three different types prepared by Boston University for this study are shown in Figures 12 and 13. The first type was a porous nickel/yttria stabilized zirconia (Ni/YSZ) cermet material. These pellet specimens are of a composition, porosity level, and thickness commonly used for SOFC anodes in an anode supported structure. For these pellets, NiO and YSZ powders were blended and pressed uniaxially in a $29 \mathrm{~mm}(11 / 8$ in.) diameter pellet die. The pressed pellets were fired in air overnight at $1300-1400^{\circ} \mathrm{C}$, and reduced in $5 \% \mathrm{H}_{2} / \mathrm{Ar}$ at $600^{\circ} \mathrm{C}$ for $1 \mathrm{hr}$. The final pellets were $1.3 \mathrm{~mm}(0.050 \mathrm{in}$.) thick with a Ni/YSZ volume ratio of $70 / 30$. The target porosity was $25-30 \%$, which is typical of fuel cell anodes.

The second type was Ni/YSZ pellets with porosity substantially greater than $30 \%$. These were prepared by a similar procedure, except that polystyrene was added as a pore former to produce the greater porosity. This condition is representative of an anode material with an unacceptably high level of porosity.

The third type was a nickel/gadolinia doped ceria (Ni/GDC) material. A pellet of this material was prepared by a similar procedure, except that $\mathrm{NiO}$ and GDC powders were used as starting 
materials. Target porosity was $25-30 \%$. This material is an alternate composition being used by some researchers in the development of IT-SOFC's (IT refers to "intermediate temperature").

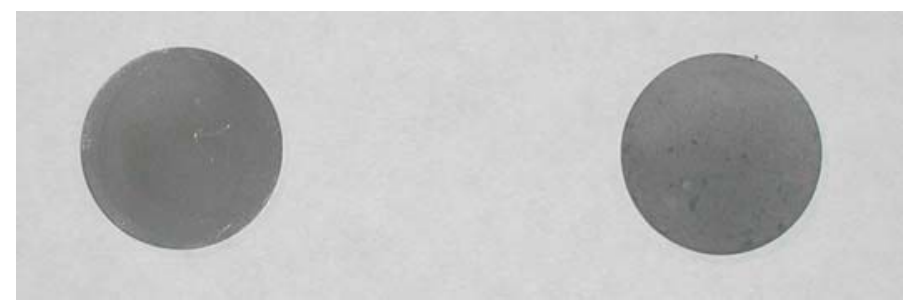

Figure 12. Pellet 3 on the left is Ni/YSZ of standard 25-30\% porosity. Pellet 1 on the right is $\mathrm{Ni} / \mathrm{YSZ}$ with substantially greater porosity. Both pellets are approximately $27 \mathrm{~mm}(1.05$ in.) dia. $\times 1.3 \mathrm{~mm}(0.05$ in.) thick.

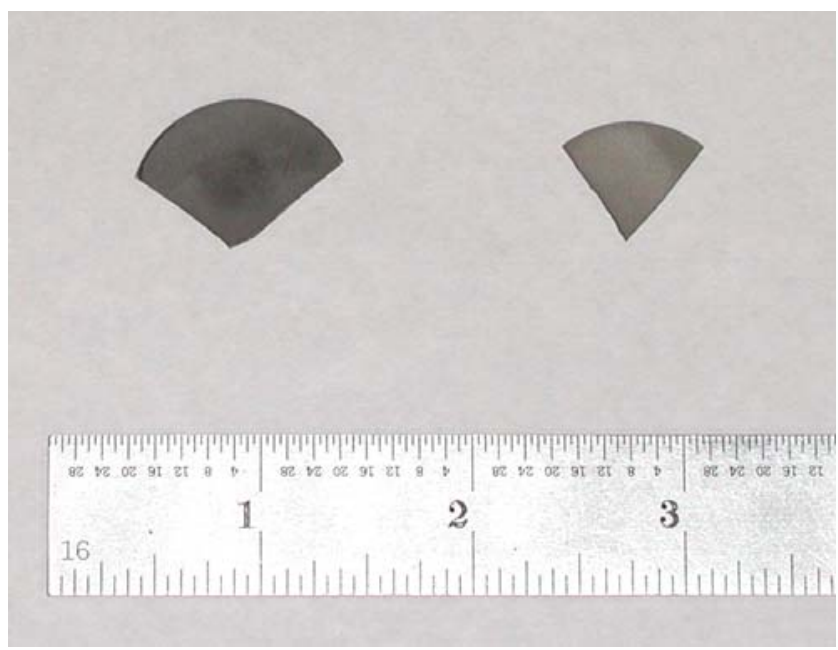

Figure 13. Two pellet sections that were also examined in this study. On the left is Ni/YSZ and on the right is Ni/GDC. Both specimens are approximately $1.3 \mathrm{~mm}(0.050 \mathrm{in}$.) thick.

\subsubsection{MWM-Array measurement procedure}

Nondestructive conductivity measurements of the pellets were performed using JENTEK's conformable FA28 MWM-Array sensor, shown in Figure 4c. This model-based eddy current sensor has 37 sensing elements that measure the local properties of interest, e.g., electrical conductivity of the pellets. The sensor continuously acquires data, and a position encoder records the location of each set of measurements as the sensor is scanned across the pellet.

For measurements on the pellet specimens in this study, grids of conductivity and lift-off (distance between the sensor and the material under test) were generated (see section 4.3.3). As the sensor was scanned with a linear encoder across the pellet, the grids were used to convert the response measured by each sensing element into property values, producing maps of conductivity and lift-off across the pellets. This grid method provides a tremendous computational speed advantage, enabling the display of property value images of the pellet virtually in real time. 
The frequencies used for MWM-Array measurements on the pellets were $6.3 \mathrm{kHz}, 20 \mathrm{kHz}$, $63 \mathrm{kHz}, 200 \mathrm{kHz}, 630 \mathrm{kHz}, 2 \mathrm{MHz}$, and $6.3 \mathrm{MHz}$. Measurements were taken with a $0.4 \mathrm{~mm}$ (15 mil) thick Mylar ${ }^{\mathbb{R}}$ shim between the sensor and the pellets. Calibration was performed by first measuring the sensor response in air and then measuring a shunted sensor response in air ("air-shunt calibration"). This calibration procedure eliminates any contribution to the response from all parts of the measurement system other than the sensing elements and obviates the need for calibration standards.

For the full-sized pellets, the FA28 sensor was mounted in the probe and cart as shown in Figure 14, and manual scans were performed by rolling the sensor/probe/cart assembly across the pellet. An encoder mounted in the front wheel of the cart records the position of each measurement.

For measuring the Ni/YSZ and Ni/GDC pellet sections, a similar procedure was used except that the sensor/probe assembly was scanned across the pellet section using a stepper motor that drew the sensor across the specimen at a uniform rate (Figure 15).

For the frequencies used in these trials, the MWM-Array sensor was measuring material response approximately $1-10$ mils $(25-250 \mu \mathrm{m})$ into the material. In future efforts, measurements at different depths could be performed using segmented field MWM-Arrays that enable sensing to different depths of penetration without altering the input current frequency (see Figure 4d).

After MWM-Array measurements on the pellets were completed, the specimens were returned to Boston University for metallographic analysis. The specimens were cut in half, polished, and examined by SEM in the secondary electron imaging mode. The porosity level in select regions of the specimens were evaluated and compared with observations made from the MWM-Array maps.

\subsubsection{Porosity mapping results and metallography}

Conductivity and lift-off images generated from scans of the Ni/YSZ pellet with the various target porosity clearly show that the conductivity varies greatly across each specimen. These variations were found on both top and bottom surfaces of the pellets. It is also clear that the conductivity of the specimen with pore former addition is substantially lower than the conductivity of the specimen with $25-30 \%$ target porosity. Note that no metallography results were available at the time of MWM-Array scanning, i.e., we were able to reach conclusions about conditions of the pellet before truth data from metallography had become available. 


\section{JENTEK Sensors Inc.}

THE ONLY SOLUTION.
110-1 Clematis Avenue, Waltham, MA 02453-7013

Phone: 781-642-9666 • Fax: 781-642-7525

email: jentek@shore.net
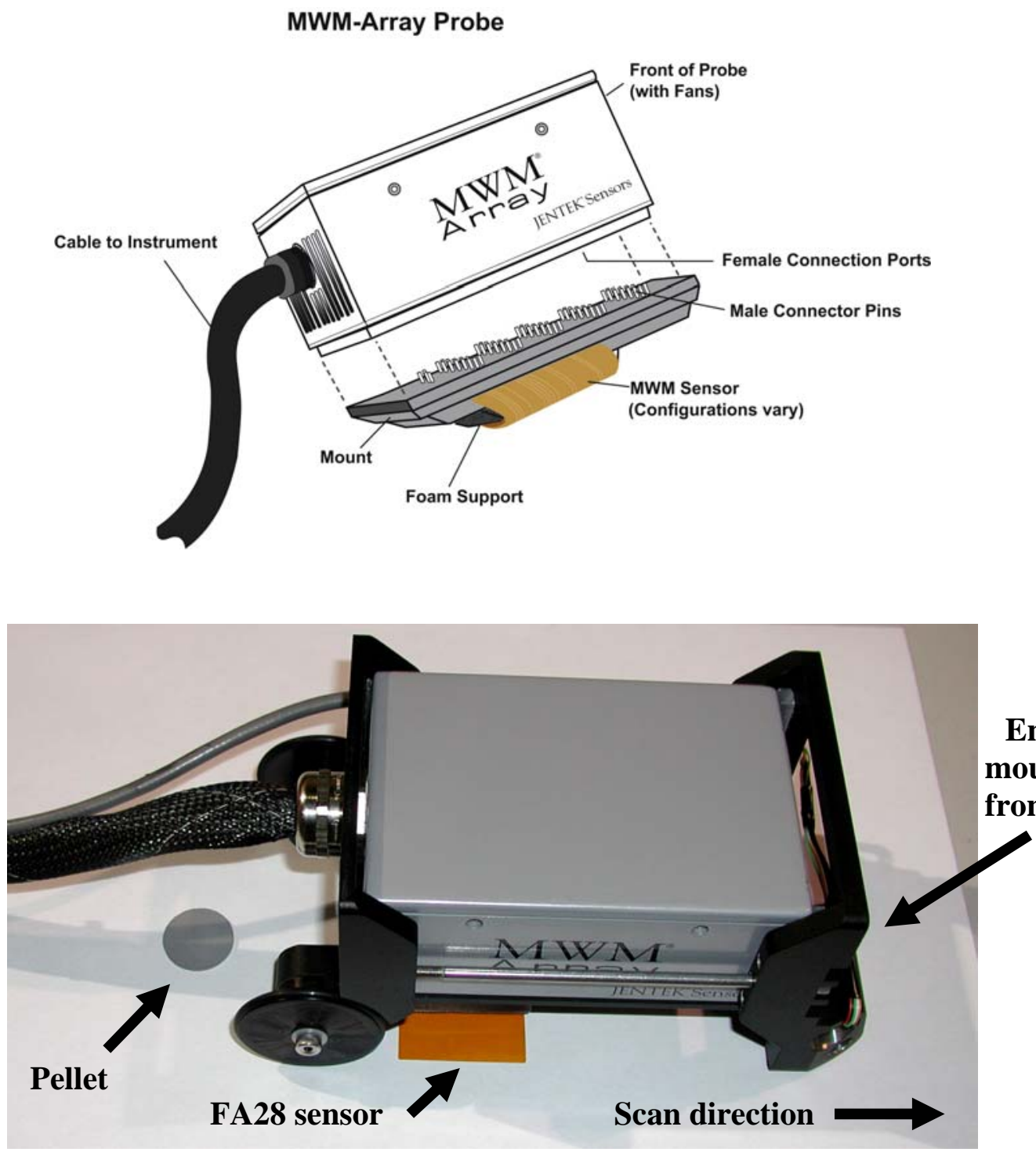

Encoder mounted in front wheel

Figure 14. Schematic of sensor/probe assembly (top), and picture of probe/sensor assembly mounted in cart (bottom). Cart was manually rolled across pellet, and encoder mounted in front wheel of cart recorded measurement position as sensor was scanned across pellet. 


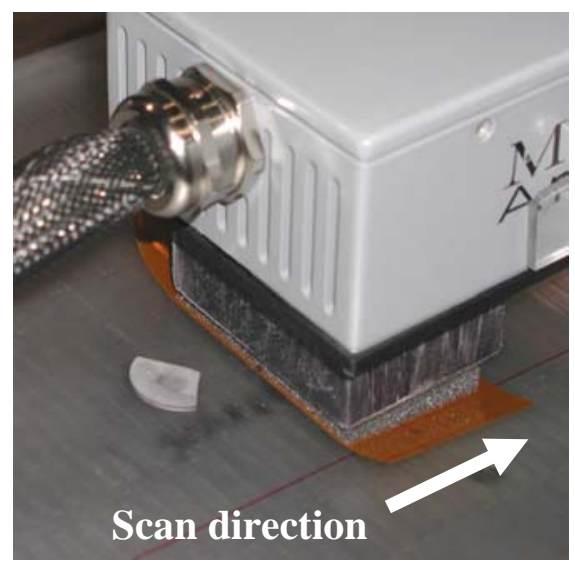

Figure 15. Scan procedure for measuring Ni/YSZ and Ni/GDC pellet sections. Probe/sensor assembly was clamped in a mechanism that translated the sensor across the specimen at a constant speed.

Metallographic analysis of these pellets confirms that the difference in conductivity corresponded to variations in porosity across the specimens. For the pellet with $25-30 \%$ target porosity, the region of high conductivity had the lowest porosity and the region with low conductivity had the highest porosity. This same correlation was found for the specimen that had pore former addition to achieve a greater target porosity. It is also clear from the SEM images that the porosity of the specimen with pore former addition was substantially greater than that of the pellet with $25-30 \%$ target porosity. Again, this is consistent with the conductivity measurement.

Scan images of the gadolinia-doped ceria (GDC) pellet section demonstrate the capability of MWM sensors to characterize both ceria-containing anodes and the YSZ-containing anodes. Again, the images show distinct differences in local conductivity across the surfaces of the pellet as well as differences in conductivity between the two sides of the pellet. The small size of this specimen resulted in some edge effects in the measurement. No metallographic analysis of this specimen was performed, though it is expected the differences in conductivity correlate with differences in porosity.

Note that the MWM-Array sensor measures local conductivity across the surface of the sample. A region of decreased conductivity could result either from increased porosity or a higher YSZ:Ni ratio. Further tests are required to determine if the measured conductivity variations were exclusively due to porosity variations or if they were also due to variations in the YSZ:Ni ratio. Note also that in these tests the sensor was measuring material response at 1-10 mils (25$250 \mu \mathrm{m}$ ) into the surface of the pellet. SEM micrographs taken of the top, middle, and bottom of the 0.050 in. $(1.3 \mathrm{~mm})$ thick pellets showed little difference in structure through the thickness of the pellet cross sections.

Subtle differences in conductivity between top and bottom surfaces of each pellet may be due to the pellet compaction step of the process, in which the powder that is in contact with the moving 
ram of the pellet die is expected to compact to a slightly higher density than the powder resting on the bottom platen.

\subsection{MWM Stress Measurements in Ferromagnetic Materials}

This section summarizes results of the Phase I effort under Task 3 which includes adaptation of MWM-Array sensors for stress measurements in the anode cermet material near the anode/electrolyte interface at ambient temperature.

\subsubsection{Overview}

The goal of this effort was to establish the feasibility of measuring stresses in Ni cermets, Ni mesh and cobalt. Previously [Zilberstein, 2002], JENTEK demonstrated a strong correlation between MWM measured magnetic permeability and stress for steels (Figure 16). The possibility of stress measurements in ferromagnetic materials is based on the inverse magnetostriction effect, or magnetomechanical effect [Bozorth, 1970]. In principle, this capability can be extended to elevated temperatures not exceeding the Curie point of the material.

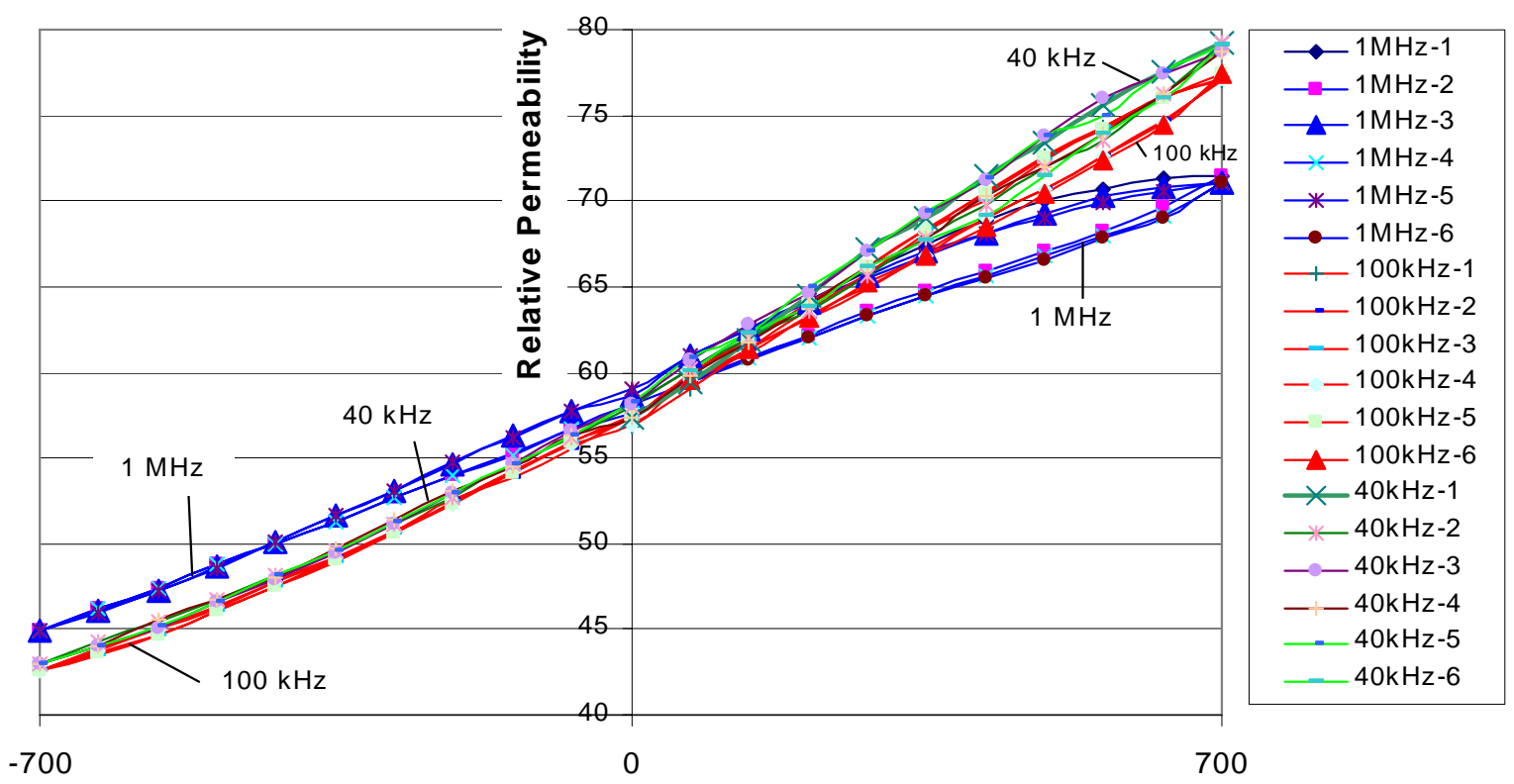

Applied Stress, MPa

Figure 16. MWM measured permeability vs. bending stress for an alloy steel specimen at stresses from -700 MPa to +700 MPa. Note that in the legend, numbers 1 through 6 for each frequency indicate the sequence of loading.

Nickel has a relatively low Curie point of $358^{\circ} \mathrm{C}$, well below the operating temperature range of SOFC stacks. This limits the temperature range over which stresses in nickel may be measured. In contrast, cobalt deposited on another material could provide the capability to monitor stress in 
the component fabricated from that material at least near the interface at SOFC operating temperatures. Moreover, for a combination of nickel and cobalt, e.g., cobalt electroplated on nickel, MWM measurements should provide a powerful combination of temperature and stress measurements, simultaneously, over a wide range of temperatures.

In this task, JENTEK demonstrated that stress changes in nickel and cobalt can be determined by measuring permeability changes with MWM sensors. JENTEK also confirmed that stress changes in a non-permeable material can be monitored if a thin layer of a permeable material is applied to it. This would allow Co-containing or Co-coated components of an SOFC to be used for monitoring of stress during operation of the fuel cell.

$\underline{\text { Materials }}$

$150 \times 150 \times 2 \mathrm{~mm}$ annealed Nickel 200 (99\% purity) sheet was purchased from Goodfellow Corporation. Two $25 \mathrm{~mm}$ wide strips were cut from this material by laser to reduce the likelihood of deformation and creation of stresses along the cut edge. The strips were used in subsequent bending tests. Information on cobalt plated specimens is provided in description of stress measurements in cobalt.

\subsubsection{Stress Measurements in Nickel}

In these tests, flat nickel specimens with dimensions of $150 \mathrm{~mm} \times 29 \mathrm{~mm} \times 2 \mathrm{~mm}$ $(6$ in. $\times 1.125$ in. $\times 0.080$ in. $)$, were loaded in pure bending to achieve surface strains between -0.000170 and +0.000170 as measured with strain gages mounted on the specimens. This is well within the elastic range (Young's modulus for nickel, E $\sim 210 \mathrm{GPa}$ or $30 \times 10^{6} \mathrm{psi}$ ), and the strain range corresponds to surface stresses between $\pm 35 \mathrm{MPa}( \pm 5 \mathrm{ksi})$. The yield strength of pure $\mathrm{Ni}$ is approximately $100 \mathrm{MPa}$ or about $15 \mathrm{ksi}$. Consequently, even repeated loading and unloading within this stress range will not lead to the development of residual stresses. MWM measurements were made on the centerline of the bend specimens in the constant moment ligament with frequencies ranging from $10 \mathrm{kHz}$ to $3.2 \mathrm{MHz}$. The experimental setup is shown in Figure 17.

The results of the MWM measurements of magnetic permeability and liftoff for the nickel specimens loaded in bending are shown in Figure 18. Ferromagnetic Ni and Ni-based alloys belong to a different class of ferromagnetic materials than steel, primarily with respect to the effect of stress on permeability [Bozorth, 1978]. The saturation magnetostriction for polycrystalline $\mathrm{Fe}$ is positive, approximately $+0.8 \times 10^{-5}$, while that of $\mathrm{Ni}$ is larger and negative, between -2.3 and $-4.7 \times 10^{-5}$. Figure 19 shows the MWM measured permeability and liftoff respectively, as a function of applied strain from -170 to $+170 \times 10^{-6}$, corresponding to a stress range from -35 to $+35 \mathrm{MPa}(-5$ to $+5 \mathrm{ksi})$. Lift-off is not expected to change when measured with MWM sensors. Lift-off variations observed at some of the frequencies are likely to be a result of an inaccurate model used in these preliminary measurements. However, the permeability measurement results obtained at frequencies yielding constant lift-off clearly show feasibility of stress measurements in nickel using MWM sensors and MWM-Arrays with grid methods. 
JENTEK Sensors Inc.

THE ONLY SOLUTION.
110-1 Clematis Avenue, Waltham, MA 02453-7013

Phone: 781-642-9666 - Fax: 781-642-7525

email: jentek@shore.net
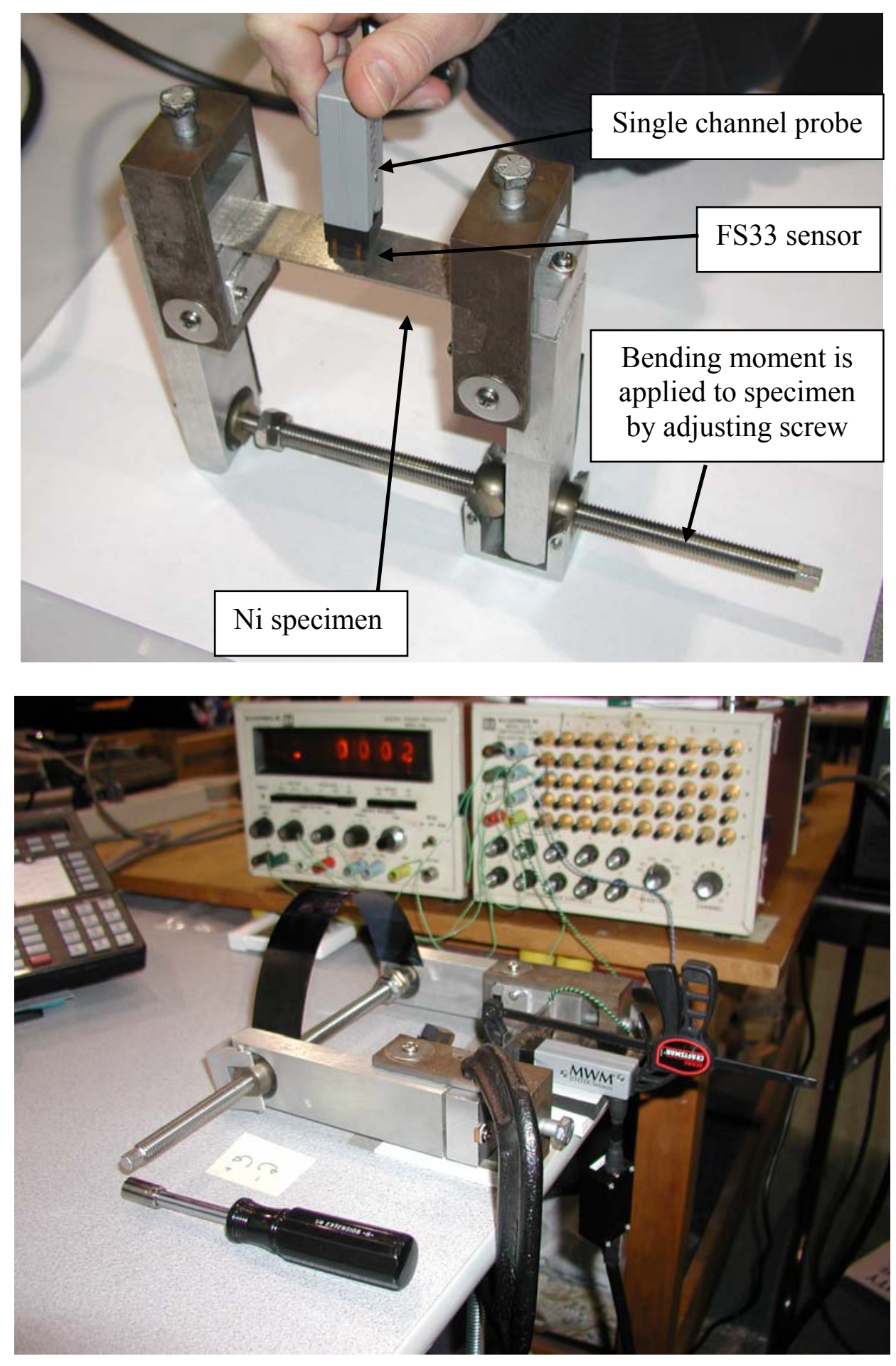

Figure 17. Experimental setup used to measure stresses in nickel via MWM magnetic permeability measurements. Simplified view of test fixture (top) and actual setup used for measurement. 

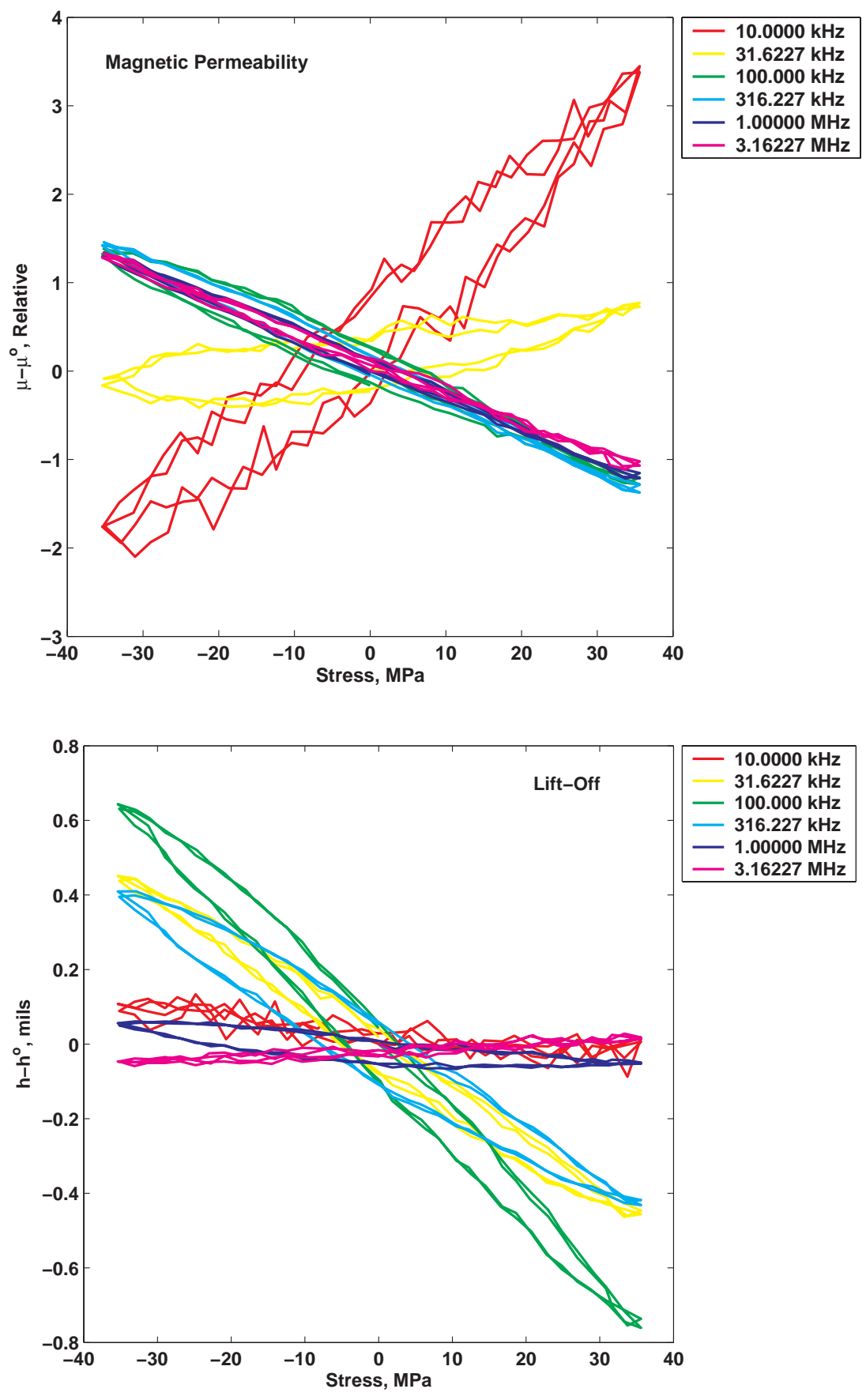

Figure 18. MWM measured permeability (top) and lift-off (bottom) measured over a range of stresses between -35 MPa (-167 $\mu$ strain) and + $35 \mathrm{MPa}(+167 \mu$ strain). This stress range is defined in terms of bending stresses at the surface. 


\subsubsection{Determination of Ni permeability and optimization of lift-off}

The general range of magnetic permeability in nickel and a suitable range of lift-off to use for the measurements were determined from tests performed on the as-received Ni plate. Measurements were made with an FS33 single-channel sensor (Figure 4b) using Mylar shims of various thicknesses between the sensor and plate to vary lift-off. Permeability/lift-off grids for these measurements were generated, assuming a conductivity of $27.8 \%$ IACS (value obtained from literature). Measurements were performed at selected frequencies in the range from $6.3 \mathrm{kHz}$ to $6.3 \mathrm{MHz}$.

Results from FS33 measurements on the nickel plate are shown in Figure 19. These plots of permeability at various frequencies and lift-offs show that the measured permeability value is dependent on frequency. It is not clear whether the differences are due to the model used or due primarily to residual stresses inadvertently introduced in presumably annealed plate (note that lower frequencies penetrate deeper into the material and could be measuring actual differences in properties). These results also show that nearly the same permeability values are obtained for lift-off values between 10 and 25 mils $(250-640 \mu \mathrm{m})$. Within this lift-off range, the measured values at each frequency differ by less than $3 \%$, demonstrating the robustness of the model-based method.
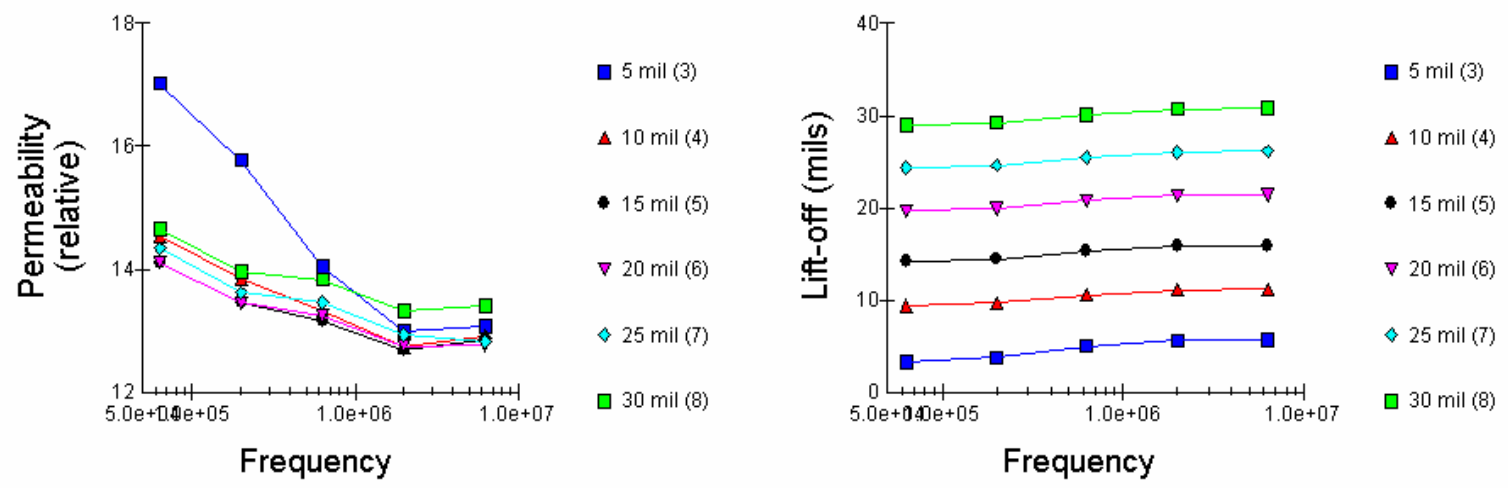

Figure 19. Measurements of nickel plate permeability and lift-off using Mylar shims of various thickness between sensor and material. Measured permeability values vary somewhat with frequency, and are relatively insensitive to differences in lift-off in the 10 to 25 mil (250 to $640 \mu \mathrm{m})$ range, varying by a maximum of $3 \%$ with lift-off across this range.

\subsubsection{Scanning Ni plate to map magnetic permeability across its surface}

Before performing measurements of magnetic permeability vs. applied stress in nickel (described on three preceding pages), the starting condition of the material was first determined. This was accomplished by scanning the Ni plate with an FA28 MWM-Array sensor to build an image of permeability across the plate. Measurements were performed at a few selected frequencies. Scans were performed after laser cutting to observe any effect on the material from the cutting operation and/or handling. The sensor and probe were mounted in a JENTEK scanning fixture, similar to the one shown in Figure 15. Since the width of the FA28 MWM-Array is $37.5 \mathrm{~mm}$ (1.48 in.), measurements from five overlapping scans were combined to generate a full image of 
the 150-mm (6-in.) wide plate. Again, the permeability/lift-off grids used for these measurements assumed a conductivity of $27.8 \% \mathrm{IACS}$.

FA28 scans of the nickel plate and strip are shown in Figure 20. It is clear from the nonuniform permeability distribution revealed by these images that the material was indeed altered when sent to the vendor for laser cutting. A visual examination of the plate indicated different surface condition due to relatively "aggressive" grinding/sanding of the surface after laser cutting. This machining operation is known to introduce residual stresses near the surface [Metals Handbook, 1989], and most likely have contributed to the permeability variations, both across the surface and with depth.

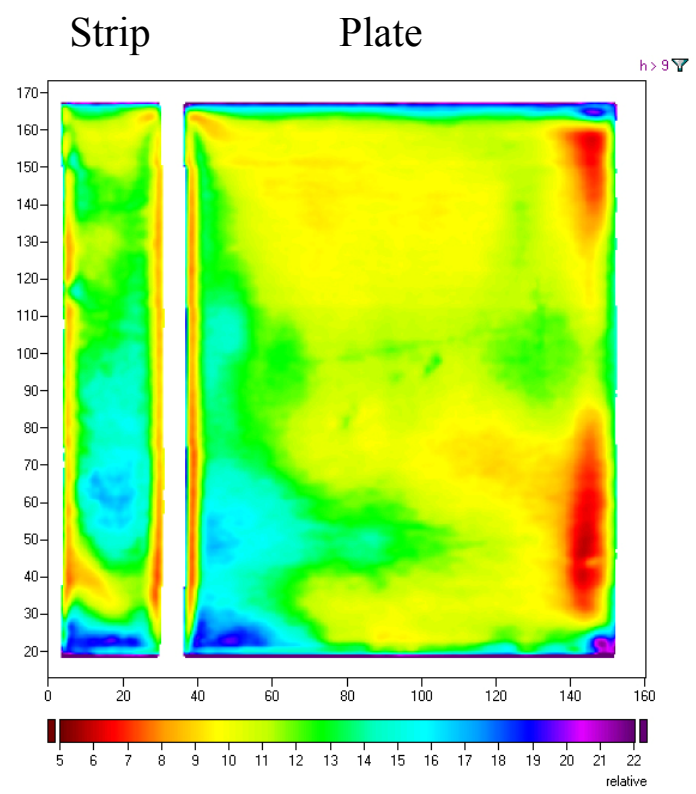

Figure 20. Scan image of nickel plate (right) and strip (left) after return from laser cutting vendor. Colors represent measured permeability values across surface of plate (refer to scale at bottom of image).

\subsubsection{Stress measurements in cobalt}

There were two reasons for measuring magnetic permeability in cobalt. The first was to confirm the expected ability of MWM sensors to detect stress changes in cobalt. Cobalt is of interest because it could be used for stress monitoring of fuel cell system components at SOFC operating temperatures. The second was to confirm that stresses in nonmagnetic components could be monitored by applying a thin layer of a ferromagnetic material. This is one of the most exciting results demonstrated in Phase I.

For these tests, $25.4 \mathrm{~mm} \times 305 \mathrm{~mm} \times 0.38 \mathrm{~mm}(1$ in. $\times 12$ in. $\times 0.025$ in. $)$ strips of brass alloy 260 were used. Strips were electroplated with cobalt on one half, i.e., $152 \mathrm{~mm}$ (6 in.) long part of one side to a thickness of $15 \mu \mathrm{m}$ (0.0006 in.). Using the equipment and procedures developed under an Air Force SBIR, three FS35 sensors (shown in Figure 21) were calibrated in air, then mounted on the plated specimen as shown in Figure 22. Total added lift-off was 14 mils, including a 10 mil Mylar shim and two adhesive layers. The cobalt-plated brass strip was 
JENTEK Sensors Inc.

THE ONLY SOLUTION.
110-1 Clematis Avenue, Waltham, MA 02453-7013

Phone: 781-642-9666 - Fax: 781-642-7525

email: jentek@shore.net

mounted at one end in a vise. A weight was affixed to the free end of the strip, and the free end was deflected and released to produce an oscillating motion in the strip. As the strip oscillated, the surface was alternately put into tension and compression. Changes in permeability associated with the changing stress state were measured with the MWM sensor, using permeability/lift-off measurement grids. The model used to construct the grids assumed three layers: nonconductive layer (lift-off), a $15-\mu \mathrm{m}$ thick cobalt layer of conductivity $25 \% \mathrm{IACS}$, and an infinitely thick brass layer of conductivity $26.5 \%$ IACS.
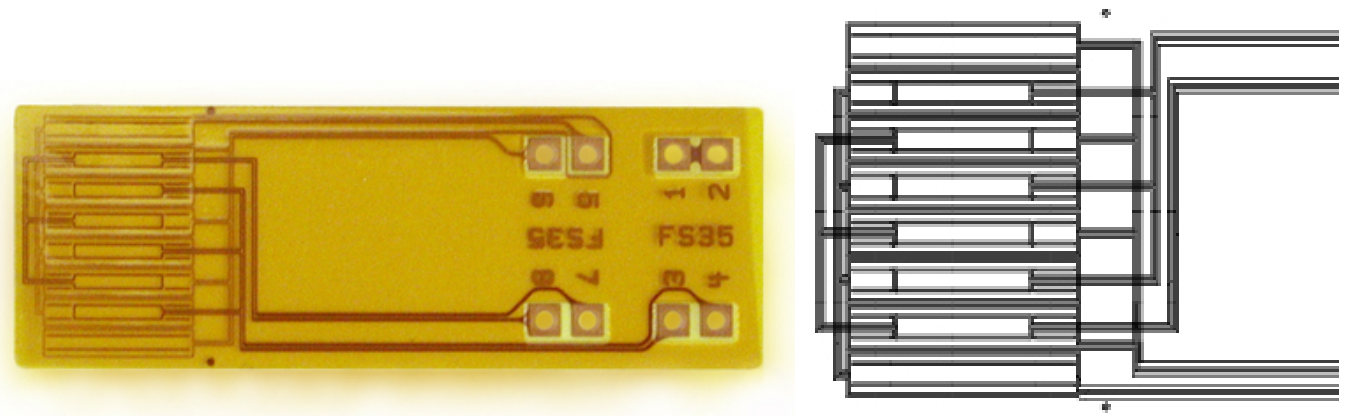

Figure 21. FS35 sensor used for stress measurements in cobalt-plated brass strip.

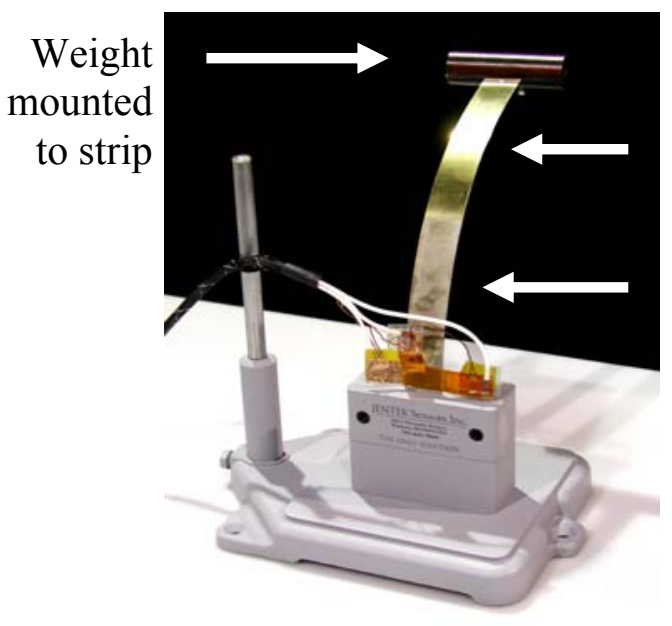

Brass strip

Cobalt plating, bottom half
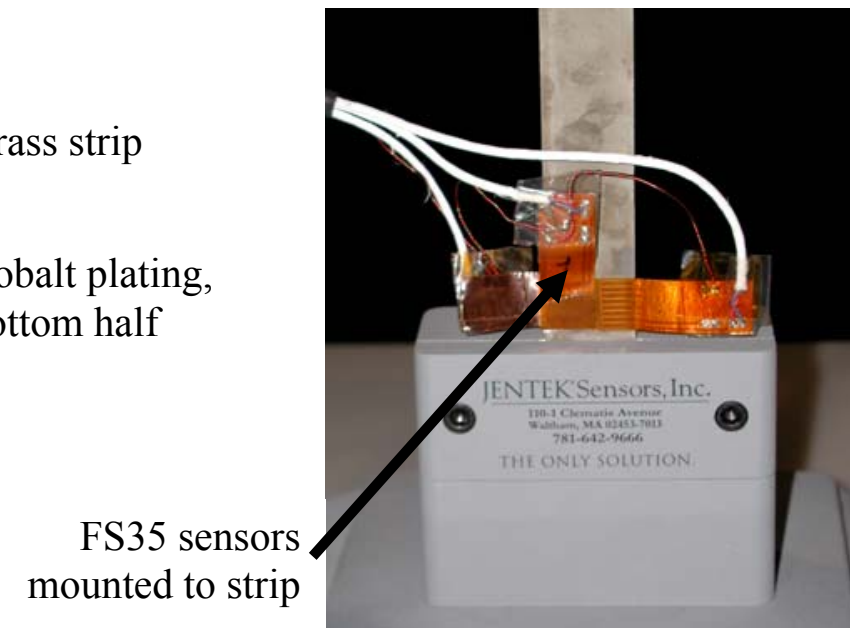

Figure 22. Fixture used to measure stress changes in cobalt-plated brass. Strip with weight attached to free end was deflected and released so that it oscillated, creating an alternating tension/compression stress condition in the strip.

Similar measurements were taken on the unplated brass as a control for the experiment. For these measurements, the strip was flipped upside down with the unplated brass end in the vise and the sensors mounted on this unplated region. Sensor response was again measured as a function of stress changes in the brass as the strip oscillated back and forth. To generate the conductivity/lift-off grids used for these measurements, the model assumed two layers:lift-off 
and an infinitely thick brass layer. This control experiment was to confirm that sensor response was not affected by artifacts such as the specimen shape change during bending or movement of the wires as the sensors oscillated back and forth with the metal strip.

No periodic response was observed when measurements were made on the unplated brass end of the strip as it oscillated back and forth. Figure 23 shows the FA35 response to the stresses created in the oscillating cobalt-plated brass strip. For this test, the strip was first deflected, then released to start the oscillations. It is clear from these measurements that the sensor is indeed sensitive to permeability changes associated with stress, even though the coating is only $15 \mu \mathrm{m}$ $(0.6$ mils $)$ thick. The reason for a slight irregularity in the permeability changes observed in the first few cycles has not been established yet.

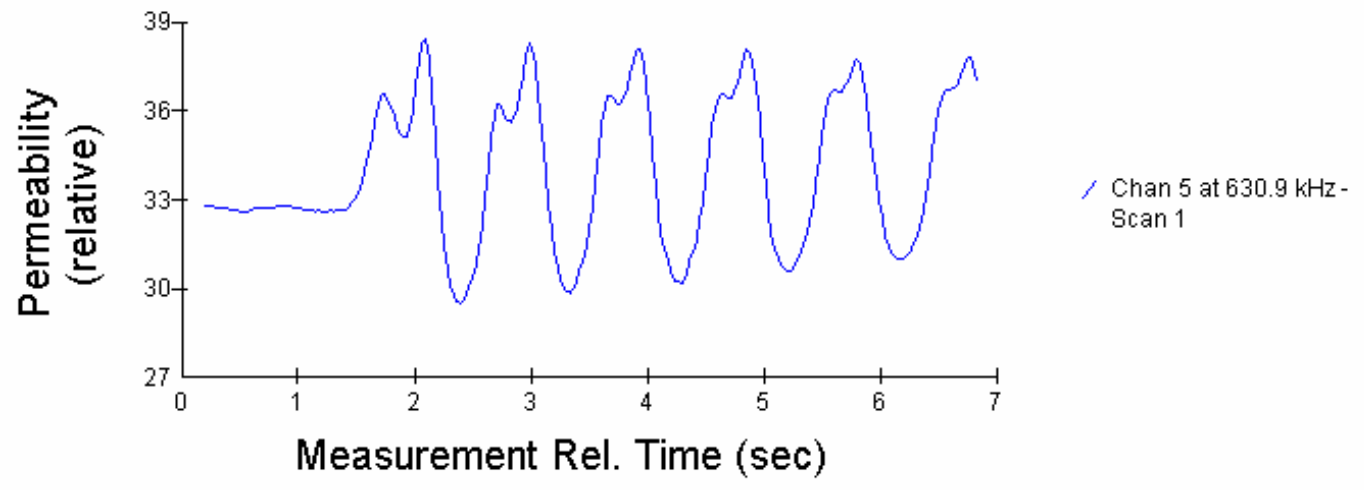

Figure 23. FS35 sensor response to the stresses created in the oscillating cobalt-plated brass strip. Note that sensor had been calibrated in air, then mounted on the strip to perform this measurement.

\subsection{Measurements on Cathode Materials}

This effort was completed under Task 4 of this Phase I SBIR. The main obstacle to measuring the properties (i.e., conductivity, density, etc.) of the LSM cathode is its relatively low electrical conductivity, especially at room temperature. Typical values for the conductivity of LSM are $0.5-1 \mathrm{kS} / \mathrm{m}$ at room temperature and $7-15 \mathrm{kS} / \mathrm{m}$ at the operating temperature, while the most sensitive conductivity range for MWM sensors and arrays is typically well above $10 \mathrm{kS} / \mathrm{m}$.

In order to assess the difficulty of carrying out such measurements, JENTEK performed simulations using JENTEK's forward model of the MWM response. The FS33 sensor was used in the simulations, because its spatial wavelength of $3.6 \mathrm{~mm}$ is large enough for working with layer thicknesses up to $2-3 \mathrm{~mm}$. 


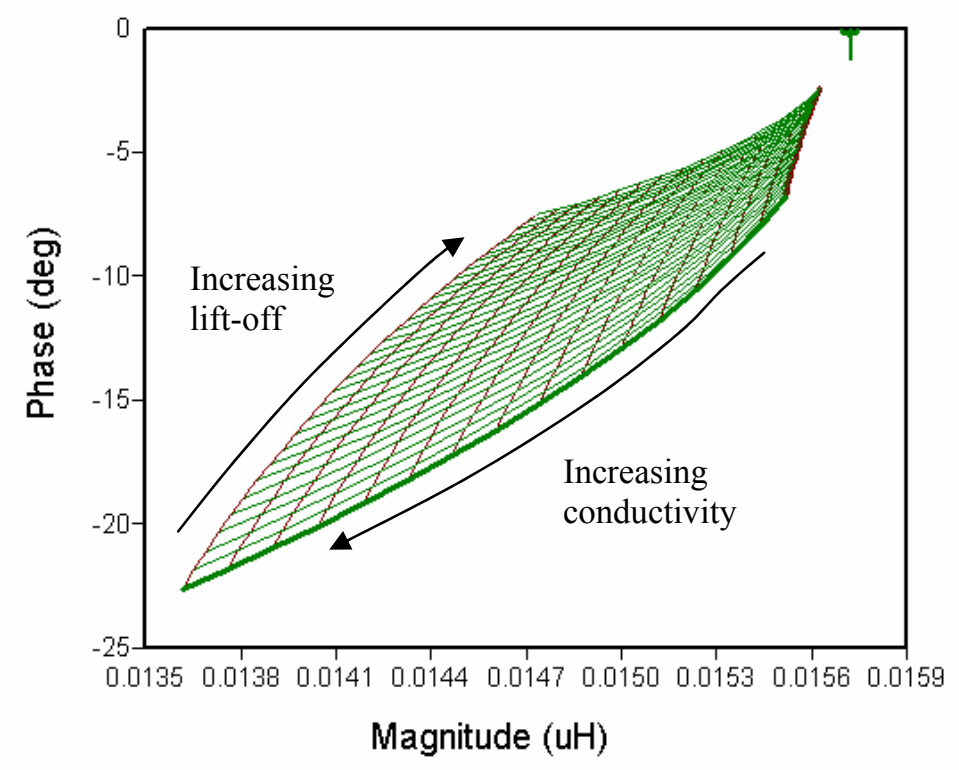

Figure 24. Conductivity/Lift-off grid at $25 \mathrm{MHz}$ for a $2 \mathrm{~mm}$ thick cathode. The conductivity range of the grid is $5-20 \mathrm{kS} / \mathrm{m}$. The conductivity of a typical LSM cathode at the operating temperature is $7-15 \mathrm{kS} / \mathrm{m}$.

Figure 24 shows a conductivity/lift-off grid, generated for these conditions. The low conductivity of the material necessitates high frequency operation, and $25 \mathrm{MHz}$ is at the top of the frequency range where the JENTEK instrumentation performs best. As can be seen in the figure, the grid range spans about 20 degrees of phase and $13 \%$ of the air point magnitude. This is considered a reasonably wide range. Furthermore, the grid is well behaved in that it has a flat topology and exhibits reasonable selectivity (selectivity is the capability to measure two unknowns, e.g., conductivity and lift-off, independently and is related to the orthogonality of the grid lines). We may therefore conclude that measuring LSM cathode conductivity at the operating temperature, for cathode-supported structures, where the cathode is on the order of $1 \mathrm{~mm}$ thick, can be expected to function well, at $25 \mathrm{MHz}$.

Since this is a high temperature measurement, the MWM sensor will have to be designed for high temperature operation, with an appropriate choice for an insulating substrate, e.g., ceramic, and an appropriate winding material.

Cathode thickness measurement under the conditions described so far should also be expected to work well. Figure 25 shows a thickness/lift-off grid that confirms this assessment. Note that for quality assessment, the temperatures of components can be raised high enough to permit accurate conductivity and thickness measurements. Also, measurements at multiple temperatures may provide useful information about cathode material condition. 


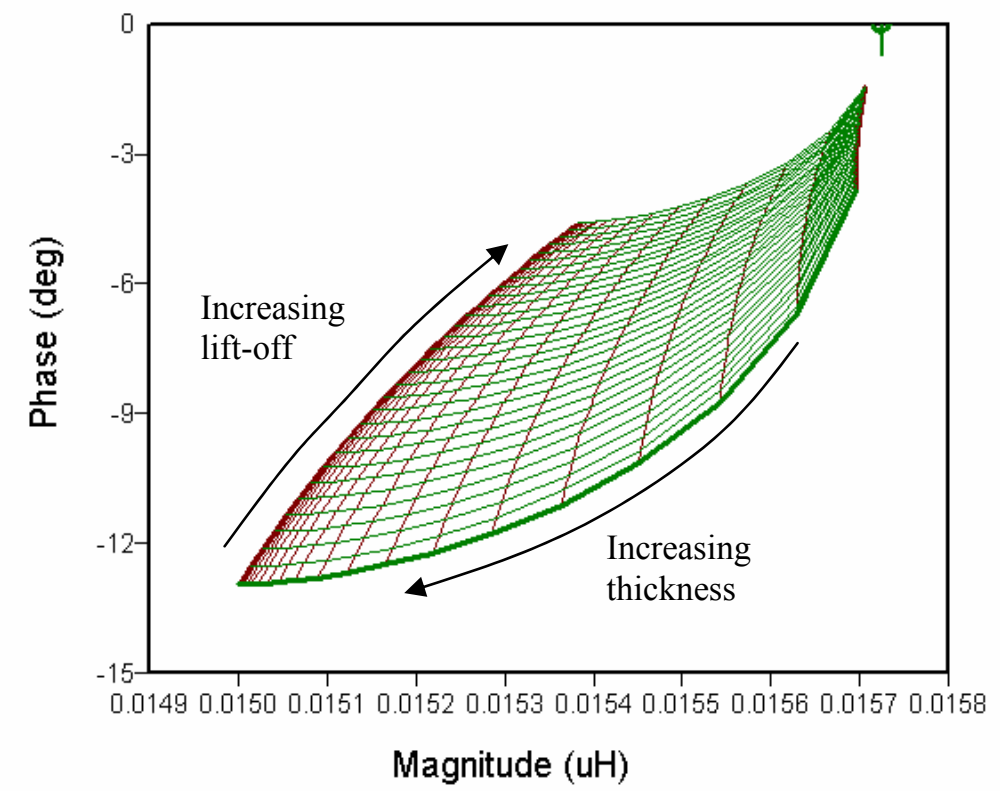

Figure 25. Thickness/Lift-off grid at $25 \mathrm{MHz}$. The conductivity of the cathode is $10 \mathrm{kS} / \mathrm{m}$. The thickness range for the grid is $0.1-3 \mathrm{~mm}$.

Inspecting the same thick (i.e., structure supporting) cathode at room temperature presents more of a challenge. A grid with the appropriate conductivity range is shown in Figure 33. Although the grid's topology and selectivity are still good, the phase range of three degrees and especially the magnitude range of under $0.25 \%$ may result in poor property estimation reliability, implying that long integration times, averaging, pick-and-place, or other noise reduction techniques may be required to achieve the required measurement quality. 


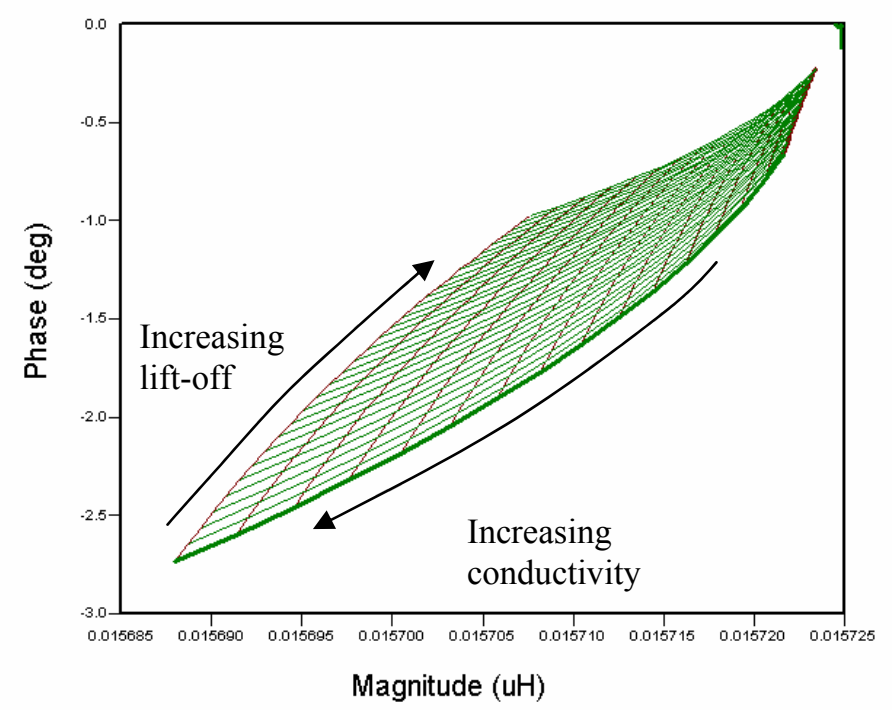

Figure 26. Conductivity/Lift-off grid at $25 \mathrm{MHz}$ for a $2 \mathrm{~mm}$ thick cathode. The conductivity range of the grid is $0.5-2 \mathrm{kS} / \mathrm{m}$. The conductivity of a typical LSM cathode at room temperature is $0.5-1 \mathrm{kS} / \mathrm{m}$.

Next, consider fuel cell stacks that are not cathode-supported, with the cathode thickness ranging between 10 and 50 microns. The sheer low volume of material in this case presents an obstacle for the induced eddy currents to generate a strong enough signal. A conductivity/lift-off grid simulating this situation at operating temperature (i.e. high conductivity) is shown in Figure 27. Its characteristics are very similar to the grid in Figure 33 (which modeled the high thickness low conductivity case), with phase range of under four degrees and magnitude range of under $0.2 \%$. In this case, we must once again conclude that serious noise reduction methods and further experimentation would be needed to achieve an acceptable level of signal-to-noise performance. Thickness measurements under these conditions would be similarly difficult, as seen in the grid in Figure 28. The correct approach here is to reduce the effective penetration depth for the sensor. This would require use of MWM winding spacing of less than 100 microns (0.004 in.), and lift-offs of less than 50 microns. 


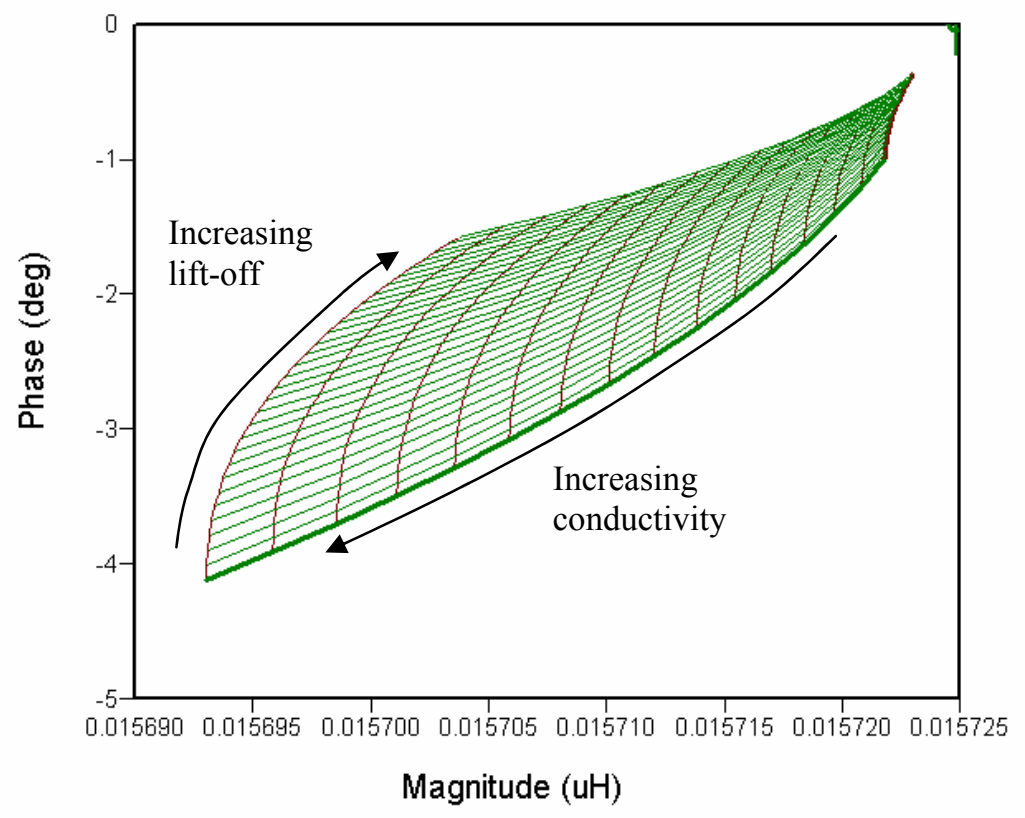

Figure 27. Conductivity/Lift-off grid at $25 \mathrm{MHz}$ for a $50 \mu \mathrm{m}$ (micron) thick cathode. The conductivity range of the grid is $5-20 \mathrm{kS} / \mathrm{m}$.

The grid shown in Figure 29 shows further that for thin layers and low temperatures the measurement will be extremely difficult. For the grid in Figure 29, the phase range is under 0.4 degrees and the magnitude range is under $0.005 \%$, which is too small to represent on the scale of the figure. The grid ranges are below the expected noise of the instrumentation. Thus, extreme measures such as long integration times and even use of differential sensing elements and through transmission over large surface areas would be required. Fortunately, these measurements could be made at much higher temperatures if practical.

The ability to accurately predict sensor performance for a given set of conditions, without having to conduct costly and time-consuming experiments, is one of the great strengths of the modelbased sensors such as JENTEK's sensors. 
JENTEK Sensors Inc.

THE ONLY SOLUTION.
110-1 Clematis Avenue, Waltham, MA 02453-7013

Phone: 781-642-9666 - Fax: 781-642-7525

email:jentek@shore.net

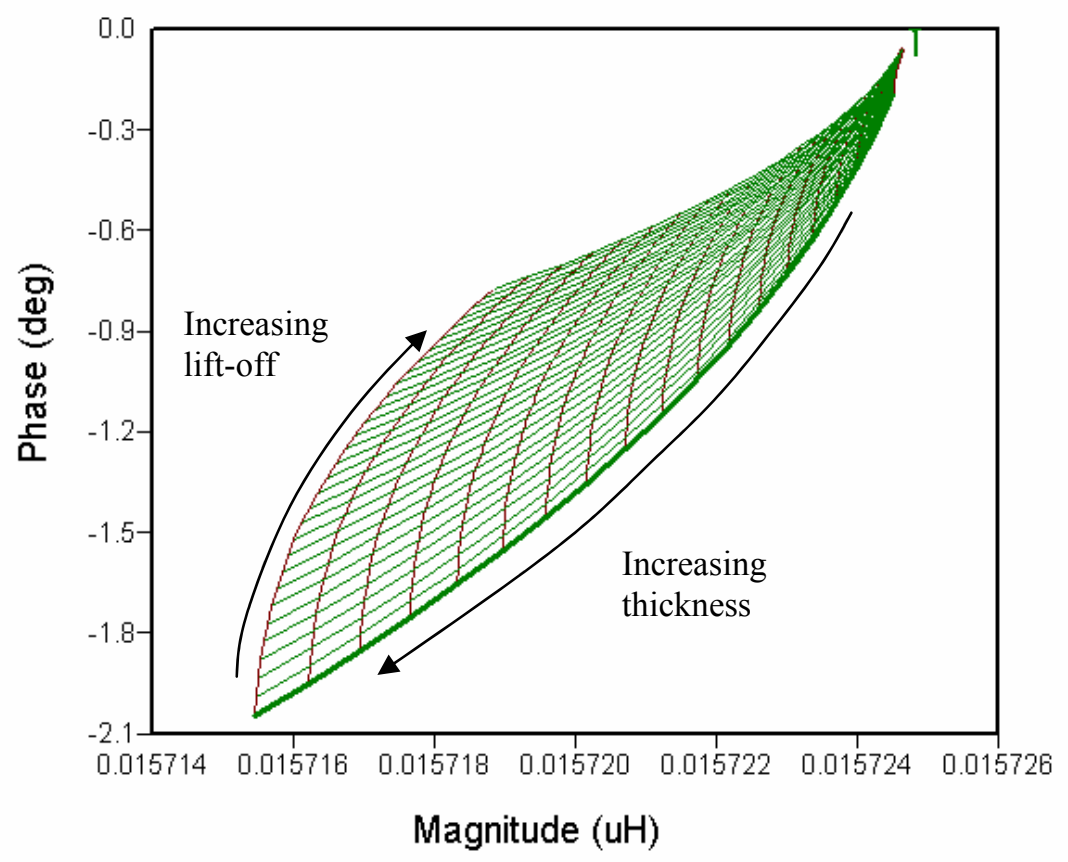

Figure 28. Thickness/Lift-off grid at $25 \mathrm{MHz}$. The conductivity of the cathode is $10 \mathrm{kS} / \mathrm{m}$. The thickness range for the grid is $\mathbf{5 - 5 0} \mu \mathrm{m}$ (microns).

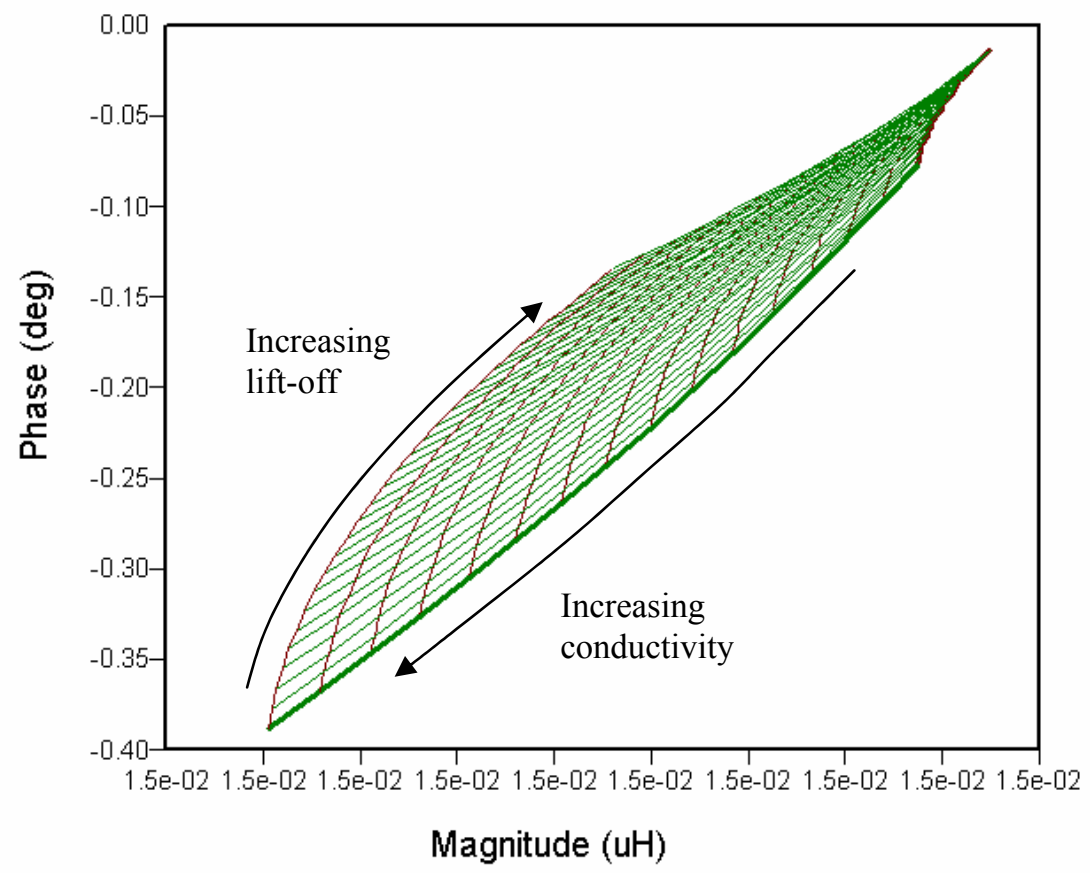

Figure 29. Conductivity/Lift-off grid at $25 \mathrm{MHz}$ for a $50 \mathrm{\mu m}$ (micron) thick cathode. The conductivity range of the grid is $0.5-2 \mathrm{kS} / \mathrm{m}$. The span of this grid is impracticably small, implying that carrying out measurements with it would be extremely difficult or impossible. 


\subsection{Identification of Another Property/Application of Interest}

This investigation was performed under Task 5 of this Phase I SBIR. In the fabrication of solid oxide fuel cells, it is expected that JENTEK's MWM inductive or IDED capacitive sensors can be used in many ways to reduce the $\$ / \mathrm{kW}$ figure of merit for stack assemblies. As described earlier, cost reductions can be achieved through identification and removal of defective cells prior to assembly into the stack. The $\$ / \mathrm{kW}$ figure can also be reduced by maximizing the power output of each cell through assurance that the full area of the cell conforms to specification, i.e., minimizing "dead zones" in the cell.

Below are examples where MWM or IDED sensors and arrays can be used to evaluate layer properties during cell fabrication. The measurements are nondestructive, and scan images provide virtually real-time information about the material properties. Note that each type of sensor, e.g., MWM or IDED can provide material characterization over a range of properties (e.g., conductivity and thickness) specific to the sensor type. Specific conditions of interest to SOFC applications would have to be tested to confirm measurement capability and to confirm that the sensitivity of the sensor and robustness of measurements match the requirements.

Example 1: 1.5-mm thick cathode-supported structure (tubular or planar design), $20 \mu \mathrm{m}$ electrolyte is deposited on cathode in the green state, co-fired, then $\mathbf{4 0} \mu \mathrm{m}$ anode is deposited and fired. IDED capacitive sensors could be used to characterize the cathode and electrolyte layers while in the green state. Using IDED-Array sensors, scan images of dielectric constant across the cathode material in the green state might identify regions of different binder/solids ratio that could later result in porosity variations. Note that the choice of IDED sensors for this application assumes the conductivity of the cathode material is sufficiently low. If it is too conductive in this state, MWM-Arrays could be used instead, in which case the scans would generate images of conductivity across the material surface. (Note: MWM-Array measurements might be made at higher temperatures to enhance sensitivity to defects and material properties.) After firing, the cathode layer becomes electrically conductive, and MWM-Arrays can be used to characterize both the cathode and electrolyte layers. Cathode conductivity and electrolyte thickness maps, measured as lift-off, could be generated simultaneously from the same scan. Here, the MWM lift-off would provide a measure of the electrolyte thickness (since its conductivity is so low it appears as a nonconducting gap to the MWM's magnetic fields at MQS frequencies). After the anode is deposited, MWM-Array scans from the anode side (in the green or fired state) could measure total anode/electrolyte thickness (i.e., as lift-off). Also, IDEDArray scans from the anode side of the structure could be used to image thickness or dielectric constant of the anode in the green or fired state.

Example 2: The cathode-supported structure in Example 1 is chemically reduced so that the NiO/YSZ anode is converted to an electrically conductive Ni/YSZ cermet. Here the full potential of JENTEK's MWM technology can be utilized to measure material properties in one, two, or possibly all three layers of the structure simultaneously. Sensor design and measurement procedures could be tailored to the specific application so that all layers contribute to the sensor response. Properties such as anode conductivity, electrolyte thickness, and cathode conductivity can be measured simultaneously, within certain conductivity and thickness values for each layer. This information could be obtained from scans performed on the anode side or the cathode side of the cell. 
Example 3: 1.5-mm thick anode-supported structure (tubular or planar design), $20 \mu \mathrm{mm}$ electrolyte is deposited on anode in the green state, co-fired, then $\mathbf{4 0 ~} \mathrm{\mu m}$ cathode is deposited and fired. IDED-Array scans of dielectric constant can be performed on the anode or anode/electrolyte material in the green state. Scan images can be used to detect inhomogeneities across the surface, and regions that are out of specification could be identified. The thickness of the electrolyte and/or anode could also be determined from these scans, possibly simultaneously using JENTEK's 3-unknown or 4-unknown algorithms. After co-firing, IDED scans could again map the thickness and dielectric constant uniformity of either or both layers, depending on the range of properties. After the cathode is deposited, it is likely that IDED sensors could no longer be used because its conductivity is too high for measurements with a dielectrometer. Also, in this example, MWM inductive sensors probably would not be used to evaluate any layer properties. The only electrically conductive layer is the cathode after it has been fired, and it is relatively thin and a poor conductor, significant advances in sensitivity would be required to adapt MWMs for this measurement.

Example 4: The anode-supported structure in Example 3 is reduced so that the NiO/YSZ anode is converted to an electrically conductive Ni/YSZ cermet. Here the MWM inductive sensors can be used to evaluate layer properties, as was demonstrated in Tasks 1 and 2. If MWM-Array scans are made from the anode side, images of porosity across the full surface of the anode can be generated. If scans are made from the cathode side, maps of anode porosity can be created equally well, and in addition total cathode/electrolyte thickness can be measured as lift-off. It is conceivable that cathode layer properties such as conductivity and thickness can also be determined from scans performed on the cathode side, though this would have to be evaluated.

\subsection{Monitoring and Control of SOFC Components with Model-based Sensors}

An investigation of the needs and requirements for monitoring and control of SOFCs and evaluation of potential sensor constructs has been successfully completed in Phase I. Three specific needs have been identified. The first is the need to identify conditions that may lead to inadequate performance, damage, or failure of the fuel cell, using networks of permanently mounted sensors at critical locations within the fuel cell. The second is the need to monitor the condition of critical components to permit condition based maintenance/replacement actions instead of maintenance and retirement based on time (this often results in substantial cost avoidance). The third is the need for enhanced observability of temperature and stress both at surfaces and subsurface within a fuel cell.

During Phase I, the feasibility of mounting magnetic and electric field versions of JENTEK's sensors at critical locations in a fuel cell was investigated. In Phase I, we have identified some candidate locations for permanently mounted MWM-Arrays for this SOFC design. These sensors might use projected magnetic fields to monitor (1) the stress on a buried interface (e.g., with a local cobalt coating used to enhance sensitivity to stress, as demonstrated in Phase I, see Section 4.3.5); (2) temperature of buried layers, which can be monitored as changes in electrical properties; (3) the degradation in properties, e.g., microstructure/porosity, resulting from 
JENTEK'Sensors Inc.

THE ONLY SOLUTION.
110-1 Clematis Avenue, Waltham, MA 02453-7013

Phone: 781-642-9666 - Fax: 781-642-7525

email: jentek@shore.net

continued operation at high temperatures; or (4) degradation caused by other process or environmental factors.

The outputs of these sensors might be monitored (1) continuously during operation, (2) during start-up or shut down, (3) during intentional excitation of various operating conditions (e.g., to enhance observability), and (4) at scheduled intervals or during outages to obtain more information than practical during normal operation.

The goal might be (1) enhancement of SOFC operational control; (2) root cause identification for failures either in real-time to prompt immediate responses, or post failure to provide forensic information to support repairs and for future failure avoidance initiatives; (3) operational adaptations to permit operation at reduced output until repair opportunities are available; (4) isolation of problems to avoid cascading failure modes; and (5) adaptive damage tolerance/life cycle management (see Figure 3) for the fuel cells and entire stack.

\subsection{Scale Up Issues}

In Phase I, JENTEK was also working on identification of scale-up issues that must be addressed to transition JENTEK solutions to OEMs and operators. Some of the relevant considerations are summarized below.

Effective SOFC stack health monitoring and controls will require inductive, capacitive and/or hybrid sensors for measurements at high temperatures. To achieve the goal of reduced life-time costs of SOFCs, new or existing sensors and arrays, either autonomous or integrated within the SOFC, must withstand operating temperatures of 800 to $1000^{\circ} \mathrm{C}$ (lower temperature limits, e.g., down to $700^{\circ} \mathrm{C}$ or even lower, in future lower temperature SOFCs). These sensors should be able to provide capabilities to measure/monitor porosity, thicknesses of layers, conductivity and degradation of interconnects, stresses, and temperature.

Under JENTEK IR\&D, a low-cost, single sensing element MWM, microfabricated with a Kapton substrate, was modified for operation in a laboratory furnace. The modification allowed prolonged operation of the sensor at temperatures up to $270^{\circ} \mathrm{C}$. Such sensors and arrays would be useful for in-service SOFC condition monitoring or control when mounted externally and performing through-wall measurements of internal media.

Also earlier under JENTEK IR\&D, another elevated temperature sensor was designed and fabricated for temperatures up to $550^{\circ} \mathrm{C}$. Improved materials and lead connections will need to be tested to increase this temperature range up to SOFC operating temperatures and allow it to be integrated into the stack itself. High-temperature inductive sensors can be used for SOFC stack health monitoring and control, while a capacitive or a hybrid inductive/capacitive sensor could be used to monitor the processing of the cell layers from the green state through fabrication.

In addition, cost of on-board electronics and portable data acquisition systems must be considered. JENTEK was recently awarded a Phase II SBIR with NAVAIR to develop such a low cost portable system for aircraft condition assessment. Such cost related scale-up issues will need to be investigated further for fuel cells. 
To perform room temperature scans on the fuel cell layers and/or final cell assembly, the proper tooling would need to be fabricated. For example, to scan the outside of a tube, a conformable model-based sensor would be selected or fabricated that wraps around the tube and cover the portion of the circumference that is of interest (e.g., the full circumference except for the interconnect). These conformable sensors can also be adapted to scan the inside of a tube.

For cells of planar design, a two-axis positioning system could carry the sensor over the fabricated layers or cell. For example, tape-cast material could be scanned as it lies on the runout table, with a positioning system that carries the sensor back and forth across the full area of the tape, either in contact with the material or at a small distance (lift-off) above it.

\subsection{Related Work}

The following capabilities of JENTEK sensors and/or measurement techniques demonstrated in other programs are relevant to some of the tasks in the Phase I effort:

- $\quad$ TBC characterization using the MWM with multivariate techniques

- Thermally grown oxide and ceramic coating thickness measurement

- Correlation of IDED measurements with permittivity models and porosity in YttriaStabilized Zirconia (YSZ) TBC specimens

- Temperature measurement of a metallic layer through another metallic layer

- Applied stress measurement in a magnetic layer through a nonmagnetic metallic layer

\subsection{SUMMARY AND CONCLUSIONS}

Results from the Phase I effort show that JENTEK's MWM technology has the potential to significantly enhance quality assessment and process control for stack layers and assemblies. This can reduce SOFC manufacturing costs by improving stack yield, which is a highly sensitive factor in electrode/electrolyte assembly (EEA) cost $(\$ / \mathrm{kW})$. In addition, the improved ability to characterize stack components enabled by MWM technology can lead to a higher power output of the stack by assuring that there are no "dead regions" in the cell. Again, this has the potential to significantly reduce EEA $\$ / \mathrm{kW}$ cost, which is also highly sensitive to power density.

In addition, JENTEK's technology has the potential to enhance health monitoring and process control during the operation of SOFC systems. MWM and IDED sensors and arrays can be permanently mounted at critical locations within a stack assembly to regularly monitor selected material properties during operation. Measurements can be used to guide decisions on when shutdowns are required for regular maintenance or to prevent larger system failures. Measurements that indicate early degradation of cell components can also be used to guide adjustments in system operating conditions to allow greater operating time between shutdowns.

Key conclusions of this Phase I effort include:

1. Rapid MWM scans can provide immediate (real-time) nondestructive characterization of porosity in layers that are electrically conductive. This was demonstrated in scans of anode 
material specimens. Local differences in electrical conductivity were found to strongly correlate with differences in porosity, as determined by subsequent metallography.

2. For layers that contain ferromagnetic materials such as nickel and/or cobalt, residual stresses can be mapped, and changes in stress can be detected. This was demonstrated by scans of a nickel plate in its as-received condition to produce magnetic permeability maps that reflect residual stress distribution. Also, MWM measurements on an externally stressed nickel plate showed significant changes in magnetic permeability with applied stresses.

3. The stress condition of a non-magnetically permeable material can be monitored by depositing a thin ferromagnetic layer onto it. This was demonstrated by measuring permeability changes in a cobalt-plated brass strip when stress was applied. The use of cobalt-based coatings may enable monitoring of stresses in the fuel cell, e.g. at IT-SOFC operating conditions because the Curie temperature for cobalt is $1121^{\circ} \mathrm{C}$, i.e., well above typical operating temperature for ITSOFCs.

4. MWM measurements provide rapid, nondestructive, virtually real-time display of property values. The immediate evaluation of selected cell layer properties enables a rapid feedback for adaptive process control and quality control improving process efficiency and saving time. Currently used quality control methods such as metallographically-determined porosity, can often take a week or longer.

5. An investigation of cathode material properties indicated that MWM sensors can be used to monitor selected properties such as cathode porosity in cathode supported structures where this layer is relatively thick (see Section 3.5).

6. An investigation of fuel cell processing methods indicated many opportunities for using MWM and IDED sensors to measure selected properties of electrode and electrolyte layers, such as thickness, conductivity, and dielectric constant (see Section 3.6).

7. Review of SOFC health monitoring issues, combined with our experience in component health monitoring and prognostics, indicated that high-temperature versions of model-based sensors have the potential to provide SOFC health monitoring capability. They could also be instrumental in SOFC process control and prognostics (see Sections 3.7 and 3.8).

\section{REFERENCES}

Auld, B.A. and Moulder, J.C., "Review of Advances in Quantitative Eddy Current Nondestructive Evaluation," Journal of Nondestructive Evaluation, vol. 18, No. 1, 1999.

ASTM Standard, E2338-04, Practice for Characterization of Coatings Using Conformable Eddy-Current Sensors Without Coating Reference Standards.

Carlson, E.J. Sriramulu, S., Teagan, P., Yang, Y., “Cost Modeling of SOFC Technology,” DOE contract DE-FC26-02NT41568, presentation of preliminary results at the First International Conference on Fuel Cell Development and Deployment, Storrs, CT, March 10, 2004. 
Goldfine, N.J., "Magnetometers for Improved Materials Characterization in Aerospace Applications," Materials Evaluation/March 1993, pp. 396-404.

Goldfine, N.J., and Zombo, P.J. (1994), "Combustion Turbine Blade Coating Characterization Using a Meandering Winding Magnetometer," Fall 1994 American Society of NDT Conference.

Goldfine, N.J., Clark, D., Lovett T., (1995), "Materials Characterization Using Model-based Meandering Winding Eddy Current Testing (MW-ET)," EPRI Topical Workshop: Electromagnetic NDE Applications in the Electric Power Industry, Charlotte, NC.

Goldfine, N.J., Washabaugh, A., Walrath K., Zombo P., and Miller R. (1998), "Conformable Eddy Current Sensors and Methods for Gas Turbine Inspection and Health Monitoring," ASM International, Gas Turbine Technology Conference, Materials Solutions '98, Rosemont, IL.

Goldfine, N.J., Schlicker, D., Sheiretov, Y., Washabaugh, A., Zilberstein, V. (2001), "Conformable Eddy-Current Sensors and Arrays for Fleet-wide Gas Turbine Component Quality Assessment," to be presented at ASME Turbo Expo Land, Sea \& Air, New Orleans, LA.

Goldfine, N.J., Zilberstein V., Schlicker D., Windoloski M., Washabaugh A., Grundy D., Walrath K., Tsukernick V., Cargill J.S., (2001) "High Throughput, Conformable Eddy-Current Sensor Arrays for Engine Disk Inspection including Detection of Cracks at Edges and in Regions with Fretting Damage," presented at NASA/FAA/DoD Conference on Aging Aircraft, Kissimmee, FL.

Goldfine, N.J., Zilberstein V., Schlicker D., Sheiretov Y., Walrath K., Washabaugh A., Van Otterloo D., "Surface Mounted Periodic Field Current Sensors for Structural Health Monitoring," presented at SPIE Conference: Smart Structures and Materials NDE for Health Monitoring and Diagnostics, Newport Beach, CA (March 2001).

Goldfine, N.J., Grundy D., Zilberstein V., Schlicker D., Shay I., Washabaugh A., Windoloski M., Fisher M., LaCivita K., Champagne V., (2002) "Corrosion Detection and Prioritization Using Scanning and Permanently Mounted MWM Eddy-Current Arrays," Tri-Service Corrosion Conference, San Antonio, TX.

Goldfine, N.J., Rhoads K.G,. Walrath K.E., Clark D.C., (1998) "Multiple Frequency Quantitative Coating Characterization," US Patent Application Number 09/191,668, November 13, 1998.

Goldfine, N.J., Zilberstein V., "Nondestructive Evaluation for Condition Based Maintenance and Prognostics \& Health Monitoring of Legacy and New Platforms," Virginia Beach, VA, April 2003.

Goldfine, N.J., Washabaugh A., Walrath K., Zombo P., Miller R., "Conformable Eddy Current Sensors and Methods for Gas Turbine Inspection and Health Monitoring," Rosemone, IL, October 1998.

Melcher, J.R., (1991), “Apparatus and Methods for Measuring Permeability and Conductivity in Materials Using Multiple Wavelength Interrogations," US Patent Number 5,015,951, May 14, 1991.

Metals Handbook (1989), Vol.16: Machining, ASM International.

Schlicker, D., Shay I., Washabaugh A., Goldfine N.J., "Capacitive Sensing Dielectrometers for Noncontact Characterization of Adhesives and Epoxies," Society Plastics Engineers (SPE), ANTEC; May 2002.

Shay, I., Zilberstein, V., Washabaugh A., Goldfine N.J., "Remote Temperature and Stress Monitoring Using Low Frequency Inductive Sensing," San Diego, CA, March 2003.

Sheiretov, Y. (I.Shay), (2001), "Deep Penetration Magnetoquasistatic Sensors," Doctoral Thesis, Massachusetts Institute of Technology, Cambridge, MA. 
JENTEK Sensors Inc.

THE ONLY SOLUTION.
110-1 Clematis Avenue, Waltham, MA 02453-7013

Phone: 781-642-9666 - Fax: 781-642-7525

email: jentek@shore.net

Zilberstein, V., Fisher, M., Grundy, D., Schlicker, D., Tsukernik, V., Vengrinovich, V., Goldfine, N., and Yentzer, T., "Residual and Applied Stress Estimation from Directional Magnetic Permeability Measurements with MWM Sensors," ASME Journal of Pressure Vessel Technology, Volume 124, pp. 375-381, August 2002.

Zilberstein, V.; Y. Sheiretov, A. Washabaugh, Y. Chen, N. Goldfine, (2000), "Applications of Spatially Periodic Field Eddy Current Sensors for Surface Layer Characterization in Metallic Alloys," presented at $27^{\text {th }}$ Annual Review of Progress in QNDE, Iowa State University, Ames, IA.

Zilberstein, V.; Schlicker, D. Walrath K., Weiss V., Goldfine N., (2001) "MWM Eddy Current Sensors for Monitoring of Crack Initiation and Growth during Fatigue Tests and In Service," International Journal of Fatigue 23, S477-S485.

Zilberstein, V.T., Lovett, T.J., Washabaugh A., Windoloski M., Goldfine N.J., (2001), "Applications for Conformable Eddy Current Sensors including High Resolution and Deep Penetration Sensor Arrays in Manufacturing and Power Generation," presented at ASME $7^{\text {th }}$ NDE Topical Conference, San Antonio, TX.

Zilberstein, Lyons R., Grundy D., Washabaugh A., Goldfine N.J., (2002), "MWM-Array Characterization and Imaging of Combustion Turbine Components," EPRI International Conference on Advances in Life Assessment and Optimization of Fossil Power Plants, Orlando, FL.

Zilberstein, V.; Fisher M., Grundy D., Schlicker D., Tsukernik V., Goldfine N.J., Yentzer T., (2002), "Residual and Applied Stress Estimation from Directional Magnetic Permeability Measurements with MWM Sensors," ASME Journal of Pressure Vessel Technology, Vol. 124, No. 3, pp. 375 - 381, August 2002. 